\title{
Dirichlet Domains for the Mostow Lattices
}

\author{
Martin Deraux
}

\section{CONTENTS}

1. Introduction

2. Complex Hyperbolic Geometry

3. Dirichlet Fundamental Domains

4. Elementary Properties of Bisectors

5. Experimental Methods

6. Dirichlet Domains for the Finite Groups

7. The Poincaré Polyhedron Theorem

8. Symmetries in the Fundamental Domain

9. List of the Main Vertices

10. The Picard Integrality Conditions

11. Varying the Parameters

Acknowledgments

References
2000 AMS Subject Classification: Primary 22E40, 32Q45, 51M20

Keywords: Complex hyperbolic geometry, Dirichlet fundamental polyhedron, nonarithmetic lattices
We describe an experimental method for studying the combinatorics of Dirichlet domains in the complex hyperbolic plane based on numerical and graphical techniques. We apply our techniques to the complex reflection groups that appear in Mostow's seminal paper on the subject and list a number of corrections to the combinatorics of the Dirichlet domains.

\section{INTRODUCTION}

Very few explicit fundamental domains for lattices acting on spaces of nonconstant curvature have been worked out, the main difficulty being the nonexistence of totally geodesic hypersurfaces. There is, of course, a general construction, due to Dirichlet, but in concrete examples the combinatorics of such domains remain elusive.

In this paper, we study this problem in the simplest symmetric space of nonconstant negative curvature, namely the complex hyperbolic plane. It is a Hermitian symmetric space, and its study involves a beautiful interplay between ideas from hyperbolic geometry and complex analysis. It is also a particularly interesting space to consider, because some lattices in its isometry group are not super-rigid (even though only a very small number of examples are known). Indeed, nonarithmetic lattices were first constructed by Mostow in [Mostow 80], and the list was expanded in [Deligne and Mostow 86] and [Mostow 86].

Mostow's original approach was to construct explicit fundamental domains for a certain class of groups generated by complex reflections (see Section 2.3). The corresponding Dirichlet domains and their combinatorics turn out to be extremely difficult to analyze, especially with the computer technology that was available in the late 1970s. In fact, using the techniques of this paper on a modern computer, it becomes relatively easy to see that some of the domains in [Mostow 80] are incorrect (we explain the main difficulties in Section 11). We point out that Mostow's main results that give a criterion for discreteness and arithmeticity (see Section 2.5), remain correct. Indeed, a number of proofs have appeared in the

(C) A K Peters, Ltd. 1058-6458/2005 \$0.50 per page Experimental Mathematics 14:4, page 467 
literature, see [Deligne and Mostow 86] or [Thurston 98], for instance. However, the proof in [Mostow 80] has a serious gap.

We find it worthwhile to list the known mistakes in [Mostow 80], as an illustration of the possible pitfalls of experimental methods in this area of mathematics. More importantly, we give detailed techniques for testing claims about the combinatorics of Dirichlet domains. For the sake of concreteness, we shall focus mainly on Mostow's examples of lattices, but our techniques clearly apply to any group given by a set of generators. Another application of our experimental methods will be presented in a subsequent paper [Deraux 05].

The use of computers is crucial throughout the analysis, and we shall not attempt to make our claims independent of the computer (even though it could quite reasonably be done). The latter was a recurrent theme in [Mostow 80], but it now seems somewhat artificial, the use of computers being much more widely accepted. Note that an intricate combination of numerical analysis and formal computations is needed in order to justify our experimental results, and we do not concentrate on that aspect in the present paper.

In order to prove discreteness rigorously, Dirichlet domains seem too rigid to be convenient. Most proofs of discreteness in the literature rely on the construction of polyhedra bounded by other kinds of hypersurfaces, avoiding the use of bisectors (see [Schwartz 01, Falbel and Koseleff 02], for instance). However, Dirichlet domains provide an important tool during the first encounter with a given complex hyperbolic group. On the one hand, if the group is not discrete, our techniques usually produce an explicit sequence that accumulates to the identity (the powers of an elliptic element of infinite order). On the other hand, assuming that the group is indeed discrete, the conjectural Dirichlet domain points to key geometric features of the group - it suggests a way to find important conjugacy classes (by finding the stabilizers of the various faces) and to determine whether or not the quotient has finite volume [Deraux 05].

In the case of the Mostow lattices, the information gathered from the Dirichlet construction can be used quite naturally to build slightly simpler fundamental domains and to prove discreteness using the Poincaré polyhedron theorem [Deraux et al. 05]. Other fundamental polyhedra in the complex hyperbolic plane, not obtained by the Dirichlet construction, have been worked out in [Falbel and Parker 05, Parker 05].

Finally, we mention that our experimental methods give invaluable insight into the very intricate properties of bisector intersections, as studied already in [Goldman 99] (see also [Phillips 92, Goldman and Parker 92]).

\section{COMPLEX HYPERBOLIC GEOMETRY}

It is a difficult problem to construct lattices in the isometry groups of symmetric spaces without using arithmetics. In spaces of constant curvature, a fairly simple class of groups is provided by reflection groups (i.e., groups generated by reflections). If the group is discrete, then the symmetric space is tiled by the connected components of the complement of the union of the mirrors of reflections in the group, each such component giving a fundamental domain for the action.

In complex hyperbolic space, the situation is much more complicated, since the mirrors of reflections have real codimension at least 2 (in fact, there are no real totally geodesic hypersurfaces). There are many isometric involutions that can play the role of reflections, but their fixed point sets have real codimension at least 2 , hence their complement is connected.

\subsection{The Complex Hyperbolic Plane}

We review some basic material on the complex hyperbolic plane (for more details, we refer the reader to [Epstein 87] or [Goldman 99]). Consider a Hermitian form $\langle\cdot, \cdot\rangle$ of signature $(2,1)$ on $\mathbb{C}^{3}$, linear in the first variable and antilinear in the second. We write $H$ for its matrix in the standard basis $\left\{e_{j}\right\}$, so that $H_{i j}=\left\langle e_{i}, e_{j}\right\rangle$. We refer to a vector $v$ as being positive, null, or negative if $\langle v, v\rangle<$ $0,=0$, or $>0$, respectively.

The group of linear isometries preserving $H$ is a noncompact group isomorphic to $U(2,1)$, and we consider its action on $P_{\mathbb{C}}^{2}$, the set of complex lines in $\mathbb{C}^{3}$. The group acting effectively is then $P U(2,1)$, and it acts transitively on the set of negative lines, i.e., the set of complex lines spanned by vectors $v$ with $\langle v, v\rangle<0$, with the stabilizer of a given line isomorphic to $U(2)$.

As a set, the complex hyperbolic plane $H_{\mathbb{C}}^{2}$ is the set of lines in $\mathbb{C}^{3}$ negative with respect to the Hermitian form (by the last paragraph, it is a homogeneous space $P U(2,1) / U(2))$. There is a natural metric, unique up to scaling, that makes the action of $P U(2,1)$ isometric. Endowed with such a metric, $H_{\mathbb{C}}^{2}$ becomes a symmetric space of $\frac{1}{4}$-pinched negative sectional curvature.

It is clear that the spaces obtained from different matrices $H$ of signature $(2,1)$ are naturally isometric (up to scaling of the metric). In an orthonormal basis, the Hermitian form can be described as

$$
\langle v, w\rangle=-v_{0} \bar{w}_{0}+v_{1} \bar{w}_{1}+v_{2} \bar{w}_{2} .
$$


Denoting by $\left(x_{0}, x_{1}, x_{2}\right)$ the corresponding homogeneous coordinates, $H_{\mathbb{C}}^{2}$ is contained in the affine chart $x_{0} \neq 0$, and in the coordinates $y_{j}=x_{j} / x_{0}$, it is simply given by the unit ball

$$
\left|y_{1}\right|^{2}+\left|y_{2}\right|^{2}<1
$$

The Hermitian form $(2-1)$ is called standard. It is convenient, however, to allow for nonstandard coordinates, as we often do in this paper.

For the metric with curvature between -1 and $-1 / 4$, the distance between two points is given by the formula

$$
\cosh \left(\frac{1}{2} d(x, y)\right)=\frac{|\langle x, y\rangle|}{\sqrt{\langle x, x\rangle\langle y, y\rangle}}
$$

Here and in what follows, with a slight abuse of notation, we denote by the same symbol a vector in $\mathbb{C}^{3}$ and the complex line that it spans (note that the right-hand side of (2-2) does not change under scaling of the vectors).

\subsection{Totally Geodesic Subspaces}

Given a positive vector $v$, its orthogonal complement $v^{\perp}$ (with respect to $H$ ) is a two-dimensional subspace on which the Hermitian form restricts to a form with signature $(1,1)$. The set of negative lines in $v^{\perp}$ is then a copy of $H_{\mathbb{C}}^{1}$, naturally isometric to the Poincaré disk. It is easily seen to be totally geodesic by exhibiting it as the fixed point set of an isometry (in fact, there is a 1-parameter family of isometries fixing it; see $(2-3)$ ).

Definition 2.1. The submanifolds of $H_{\mathbb{C}}^{2}$ given by $v^{\perp}$ are called complex geodesics. The vector $v$ is called polar to the complex geodesic $v^{\perp}$.

Here, we abuse notation once again, denoting by $v^{\perp}$ the set of negative lines in $\mathbb{C}^{3}$ spanned by vectors orthogonal to $v$ with respect to $H$. Let $\zeta$ be any complex number of absolute value 1 , and consider the linear map

$$
R_{v, \zeta}: x \mapsto x+(\zeta-1) \frac{\langle x, v\rangle}{\langle v, v\rangle} v
$$

It is easy to check that it preserves the Hermitian inner product, that it fixes the vectors in $v^{\perp}$, and that $v$ gets multiplied by $\zeta$. Geometrically, $R_{v, \zeta}$ fixes $v^{\perp}$ and rotates about it by the angle given by the argument of $\zeta$.

Definition 2.2. The transformation $R_{v, \zeta}$ is called a complex reflection with mirror $v^{\perp}$.
It is apparent from Equation (2-3) that the reflection remains unchanged under scaling of the vector $v$. In particular, we may assume that $\langle v, v\rangle=1$ (in what follows, we will always use this normalization).

Observe also that we may compose the above linear transformation by multiplication by a fixed scalar, without changing the corresponding isometry (scalar matrices act as the identity on projective space). A complex reflection is an isometry that can be lifted to a linear transformation of the form in $(2-3)$.

Another type of totally geodesic subspace is given in the standard ball model as the set of points with real coordinates, which is the fixed point set of the isometry $\left(y_{1}, y_{2}\right) \mapsto\left(\bar{y}_{1}, \bar{y}_{2}\right)$. In general, for a Hermitian form that is not necessarily standard, one gets such a subspace by taking three independent vectors $v_{1}, v_{2}, v_{3}$ with $\left\langle v_{i}, v_{j}\right\rangle \in$ $\mathbb{R}$ for any $i, j$. The restriction of the Hermitian form to the real span of these vectors is then a quadratic form of signature $(2,1)$, and the image in projective space of the set of negative vectors in the real span is simply a copy of $H_{\mathbb{R}}^{2}$. There is a unique isometry that fixes it, given in the basis of the $v_{j}$ by

$$
\sum \lambda_{j} v_{j} \mapsto \sum \bar{\lambda}_{j} v_{j}
$$

Such a subspace is called a totally real totally geodesic subspace, or simply a Lagrangian.

It is a fact that all totally geodesic submanifolds of $H_{\mathbb{C}}^{2}$ are points, real geodesics, complex geodesics, or Lagrangians (for a sketch of the proof, see [Goldman 99]). It can also be checked that the above two types of submanifolds realize the extrema of sectional curvature (the complex geodesics being four times more curved than the totally real planes).

Finally, we mention that $P U(2,1)$ is the group of holomorphic isometries of $H_{\mathbb{C}}^{2}$ and has index 2 in the full group $\widehat{P U}(2,1)$ of isometries. The latter is generated by $P U(2,1)$ and one antiholomorphic involution as in $(2-4)$.

\subsection{Complex Reflection Groups}

A natural way to describe a reflection group is to give a Coxeter diagram having one node for each generator and joining two nodes to mean that these generators should satisfy some braid relation [Coxeter 67].

The main difference from the real hyperbolic case is that such a diagram does not determine the group uniquely - there is one degree of freedom attached to each loop in the diagram (the so-called phase shift). A slightly more general discussion of Coxeter diagrams is given in [Mostow 80]; here, we simply review Mostow's 
examples, given by the following diagram (the meaning of the phase shift $\varphi$ will be explained below).

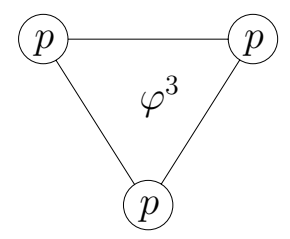

Here, there are three generators of order $p$ that satisfy the braid relation

$$
R_{i} R_{j} R_{i}=R_{j} R_{i} R_{j}
$$

We leave it to the reader to verify the following important result.

Proposition 2.3. Let $R_{1}=R_{v_{1}, \zeta_{1}}$ and $R_{2}=R_{v_{2}, \zeta_{2}}$ be distinct complex reflections as in (2-3). Then, $R_{1} R_{2} R_{1}=$ $R_{2} R_{1} R_{2}$ if and only if $\zeta_{1}=\zeta_{2}$ and

$$
\frac{\left|\left\langle v_{1}, v_{2}\right\rangle\right|}{\sqrt{\left\langle v_{1}, v_{1}\right\rangle\left\langle v_{2}, v_{2}\right\rangle}}=\frac{1}{\left|\zeta_{j}-1\right|} .
$$

Note that the left-hand side of $(2-5)$ is the cosine of the angle between the mirrors $v_{1}^{\perp}$ and $v_{2}^{\perp}$. When $\zeta=e^{2 \pi i / p}$, the right-hand side can be written as

$$
\frac{1}{2 \sin \frac{\pi}{p}}
$$

We see that there is a one parameter family of possible configurations of mirrors of the generators by the following rough dimension count: the space of triples of vectors has dimension 12 , the group $P U(2,1)$ has dimension 8 , so the configuration space of polar vectors has dimension 4 . The braiding of the generators imposes the three angles between the mirrors of the generators, so we get only one free parameter. More precisely, we have Proposition 2.4.

Proposition 2.4. The space of configurations of triples of (distinct) braiding complex reflections of order $p$, up to conjugation by an element of $P U(2,1)$, is an interval.

Proof: Let $R_{j}=R_{v_{j}, \zeta}$ (where $\zeta=e^{2 \pi i / p}$ ) be the three braiding reflections. By scaling the vectors $v_{j}$, we may assume that $\left\langle v_{i}, v_{i}\right\rangle=1$.

The braiding imposes $\left|\left\langle v_{i}, v_{j}\right\rangle\right|$, but we are still free to choose the argument of $\left\langle v_{i}, v_{j}\right\rangle$. By adjusting $v_{1}$ and $v_{3}$ (scaling them by a complex number of norm 1 ), we may assume that $\left\langle v_{1}, v_{2}\right\rangle=\left\langle v_{2}, v_{3}\right\rangle=1$.
There is one parameter left, namely the argument of the complex number $\left\langle v_{3}, v_{1}\right\rangle$. Two configurations are equivalent under conjugation by $P U(2,1)$ if and only if, after the above normalizations, we get the same argument for $\left\langle v_{3}, v_{1}\right\rangle$.

In particular, if the configurations are equivalent, then they have the same Hermitian triple product

$$
\left\langle v_{1}, v_{2}\right\rangle\left\langle v_{2}, v_{3}\right\rangle\left\langle v_{3}, v_{1}\right\rangle
$$

and it is easily checked that this is also a sufficient condition.

Definition 2.5. The quantity $\left\langle v_{1}, v_{2}\right\rangle\left\langle v_{2}, v_{3}\right\rangle\left\langle v_{3}, v_{1}\right\rangle$ is called the Hermitian triple product of $v_{1}, v_{2}$, and $v_{3}$, and it is denoted by $\left\langle v_{1}, v_{2}, v_{3}\right\rangle$.

A more symmetric way to normalize the inner products is to take $\left\langle v_{1}, v_{2}\right\rangle=\left\langle v_{2}, v_{3}\right\rangle=\left\langle v_{3}, v_{1}\right\rangle$. We then denote their common value by $-\alpha \varphi$, where $\alpha=$ $1 /(2 \sin \pi / p)$.

Definition 2.6. The complex number $\varphi^{3}$ such that $\left\langle v_{1}, v_{2}\right\rangle\left\langle v_{2}, v_{3}\right\rangle\left\langle v_{3}, v_{1}\right\rangle=-\alpha^{3} \varphi^{3}$ is called the phase shift of the configuration.

Since $\varphi^{3}$ is a complex number of norm 1, we refer to it only by its argument, and when it causes no confusion we call $\arg \varphi^{3}$ the phase shift as well.

It turns out that the phase shift cannot be chosen arbitrarily. To see this, let us first assume that the vectors $v_{j}$ are linearly independent in $\mathbb{C}^{3}$. Consider the matrix of the Hermitian form in the basis $\left\{v_{1}, v_{2}, v_{3}\right\}$, normalized in a symmetric fashion as above:

$$
H=\left[\begin{array}{ccc}
1 & -\alpha \varphi & -\alpha \bar{\varphi} \\
-\alpha \bar{\varphi} & 1 & -\alpha \varphi \\
-\alpha \varphi & -\alpha \bar{\varphi} & 1
\end{array}\right]
$$

There exists a configuration with phase shift $\varphi$ if and only if $H$ has signature $(2,1)$. In turn, this is equivalent to $\operatorname{det}(H)<0$ ( $H$ is clearly not negative-definite).

We have

$$
\operatorname{det}(H)=1-3 \alpha^{2}-\alpha^{3}\left(\varphi^{3}+\bar{\varphi}^{3}\right),
$$

which is negative for $\left.\frac{1}{\pi} \arg \left(\varphi^{3}\right) \in\right]-t_{0}, t_{0}\left[\right.$, where $t_{0}=$ $3\left(\frac{1}{2}-\frac{1}{p}\right)$.

The case when the vectors $v_{j}$ are dependent is slightly more complicated. It can be seen that it corresponds to the endpoints of the above intervals, namely to $t= \pm t_{0}$.

Recall that we assume that the generators are distinct, so the span of any two vectors $v_{j}$ is two-dimensional. We 
may choose $v_{1}$ and $v_{2}$ so that $\left\langle v_{1}, v_{2}\right\rangle=\alpha$, and take the third vector to be of the form $v_{3}=\lambda\left(v_{1}+\theta v_{2}\right)$ for $\lambda \in \mathbb{R}$ and $|\theta|=1$. The braid relation can then be checked to impose $\lambda=1$ and $\theta=i \eta$ or $\theta=-i \bar{\eta}$, where we write

$$
\eta=e^{i \pi / p}
$$

Then, the triple Hermitian product $\left\langle v_{1}, v_{2}, v_{3}\right\rangle$ is checked to be either $\alpha^{3} i \bar{\eta}^{3}$ or its complex conjugate $-\alpha^{3} i \eta^{3}$ (to verify this, it is useful to note that $1 / \alpha=-i(\eta-\bar{\eta}))$. The last two values correspond to the phase shift at the points where $t= \pm t_{0}$.

Note that in this case, the mirrors or the three generators all fix a common point represented by a vector orthogonal to any two of the vectors $v_{j}$.

In Mostow's notation, the group $\Gamma(p, t)$ is generated by three reflections as in Proposition 2.4, with phase shift

$$
\varphi^{3}=e^{\pi i t}
$$

We shall always assume that the phase shift satisfies the condition

$$
|t|<3\left(\frac{1}{2}-\frac{1}{p}\right)
$$

for the reasons stated in the proof of Proposition 2.4.

Moreover, in order to keep the notation as symmetric as possible, we shall use as homogeneous coordinates the ones given in the basis $v_{1}, v_{2}, v_{3}$ of vectors polar to the mirrors of the generating reflections. In particular, the Hermitian form is given by the matrix (2-7). Note also that in these coordinates, the symmetry of order 3 conjugating $R_{i}$ into $R_{i+1}$ (indices taken mod 3 ) is simply given by

$$
J=\left[\begin{array}{lll}
0 & 0 & 1 \\
1 & 0 & 0 \\
0 & 1 & 0
\end{array}\right]
$$

As above, we write $\eta=e^{\pi i / p}$, so that the reflections $R_{j}=R_{v_{j}, \eta^{2}}$.

The matrix of $R_{1}$ is given by

$$
R_{1}=\left[\begin{array}{ccc}
\eta^{2} & -\eta i \bar{\varphi} & -\eta i \varphi \\
0 & 1 & 0 \\
0 & 0 & 1
\end{array}\right]
$$

and the other generators are easily obtained, since $R_{2}=$ $J R_{1} J^{-1}$ and $R_{3}=J^{-1} R_{2} J$.

\section{$2.4 \quad$ Finite Groups}

Two of the generators $R_{i}$ and $R_{j}$ have common fixed point in the ball

$$
\begin{aligned}
& \Leftrightarrow \quad \exists v \in e_{i}^{\perp} \cap e_{j}^{\perp},\langle v, v\rangle<0 \\
& \Leftrightarrow \quad \operatorname{Span}_{\mathbb{C}}\left\{e_{i}, e_{j}\right\} \text { is a positive definite subspace } \\
& \Leftrightarrow \quad \operatorname{det}\left[\begin{array}{cc}
\left\langle e_{i}, e_{i}\right\rangle & \left\langle e_{i}, e_{j}\right\rangle \\
\left\langle e_{j}, e_{i}\right\rangle & \left\langle e_{j}, e_{j}\right\rangle
\end{array}\right]<0 \\
& \Leftrightarrow \quad\left|\left\langle e_{i}, e_{j}\right\rangle\right|<1 \\
& \Leftrightarrow \quad p<6
\end{aligned}
$$

where in the last equivalence we use $(2-6)$. Here, we focus on the case $p<6$, and denote by $p_{i j}$ the point common to the mirrors of $R_{i}$ and $R_{j}$ (coordinates for that point are given in Section 9).

If $p<6$, the group $\Gamma_{i j}$ generated by $R_{i}$ and $R_{j}$ can be seen as a subgroup of $U(2)$. A nice description of the group is obtained by studying its action on the complex projective line $P_{\mathbb{C}}^{1} \simeq S^{2}$ of complex lines through $p_{i j}$ (in homogeneous coordinates this corresponds to the action on the positive definite subspace $p_{i j}^{\perp}$ of $\mathbb{C}^{3}$ ).

The generating reflections act as rotations by an angle $2 \pi / p$ with centers at the vertices of an equilateral triangle with angles $2 \pi / p$. These two spherical isometries are readily seen to generate the spherical triangle group $T_{2,3, p}$. For integers $p, q, r \geq 2$ with $1 / p+1 / q+1 / r>1$, we denote by $T_{p, q, r}$ the triangle group generated by rotations around the vertices of the spherical triangle with angles $\pi / p, \pi / q$, and $\pi / r$. For $p=3,4$, or $5, T_{2,3, p}$ is the group of orientation preserving autorphisms of the regular tetrahedron, cube, or dodecahedron, which have order 12,24 , and 60, respectively.

Finally, the group $\Gamma_{i j}$ is a central extension of the triangle group $T_{2,3, p}$ (which is obtained by projectivization of the action on $p_{i j}^{\perp}$ ). The center of $\Gamma_{i j}$ is generated by the appropriate power of $R_{i} R_{j}$ and has order 2,4 , and 10 in the cases $p=3, p=4$, and $p=5$, respectively. In particular, $\Gamma_{i j}$ has order 24,96 or 600 for $p=3$, 4 , or 5 respectively.

We shall see in Section 8 that the obvious relations (i.e., the order of the reflections and the braid relation) actually give a presentation for the finite group.

Finally, we mention that the center of mass of the three mirrors of the generating reflections, which is also the center of mass of the fixed points $p_{i j}$, is given by the point

$$
p_{0}=[1,1,1]^{T} .
$$

Note that this is the isolated fixed point of the symmetry $J$. 


\begin{tabular}{|l|l|l|l|}
\hline$p$ & $|t|<\frac{1}{2}-\frac{1}{p}$ & $|t|=\frac{1}{2}-\frac{1}{p}$ & $|t|>\frac{1}{2}-\frac{1}{p}$ \\
\hline 3 & $0, \frac{\mathbf{1}}{\mathbf{3 0}}, \frac{1}{18}, \frac{\mathbf{1}}{\mathbf{1 2}}, \frac{\mathbf{5}}{\mathbf{4 2}}$ & $\frac{1}{6}$ & $\frac{7}{30}, \frac{1}{3}$ \\
4 & $0, \frac{\mathbf{1}}{\mathbf{1 2}}, \frac{\mathbf{3}}{\mathbf{2 0}}$ & $\frac{1}{4}$ & $\frac{5}{12}$ \\
5 & $\frac{1}{10}, \frac{\mathbf{1}}{\mathbf{5}}$ & & $\frac{11}{30}, \frac{7}{10}$ \\
\hline
\end{tabular}

TABLE 1. Pairs $(p, t)$ for which the conditions of the Poincaré theorem hold. Numbers in boldface yield nonarithmetic lattices.

\subsection{Mostow's Main Results}

We review the two main results of [Mostow 80].

Theorem 2.7. If $\left(\frac{1}{4}-\frac{1}{2 p} \pm \frac{t}{2}\right)^{-1} \in \mathbb{Z} \cup\{ \pm \infty\}$, then $\Gamma(p, t)$ is a lattice in $P U(2,1)$.

The above conditions come out naturally by studying the cycles of 2 -faces of the Dirichlet domains, in the terminology of the Poincaré polyhedron theorem (see Section 7). Indeed, certain cycle transformations are complex reflections that rotate around the appropriate 2 -face by angles $2 \pi\left(\frac{1}{4}-\frac{1}{2 p} \pm \frac{t}{2}\right)$ (see Section 10 ).

There are only finitely many values of $(p, t)$ that satisfy the condition in Theorem 2.7 (see Table 1). The condition of Theorem 2.7 is almost necessary for discreteness, and, in fact, it follows from the work in [Mostow 88] that there is only one more discrete group among the groups $\Gamma(p, t)$ with $p=3,4$, or 5 , namely $\Gamma\left(5, \frac{1}{2}\right)$. In that case, the Dirichlet polyhedron can be divided as a union of fundamental domains by considering the finite group that fixes $p_{0}$ (we shall not discuss this issue).

One gets more groups (but still finitely many) by allowing $p>5$, but for the sake of brevity, we shall not discuss this here (that issue and much more is treated in the various papers by Mostow and coauthors given in the bibliography, where the groups are described in terms of monodromy groups of hypergeometric functions). Some of the groups for $p>5$ are also studied in [Parker 05].

As mentioned before, the lattices of Theorem 2.7 are particularly interesting because of the following:

Theorem 2.8. The lattices $\Gamma(p, t)$ are nonarithmetic for $(p, t)=\left(3, \frac{1}{30}\right),\left(3, \frac{1}{12}\right),\left(3, \frac{5}{42}\right),\left(4, \frac{1}{12}\right),\left(4, \frac{3}{20}\right)$, or $\left(5, \frac{1}{5}\right)$.

For the definition of arithmeticity, see [Mostow 80, Deligne and Mostow 86].

\section{DIRICHLET FUNDAMENTAL DOMAINS}

Given any group $\Gamma$ acting on $H_{\mathbb{C}}^{2}$, the Dirichlet domain centered at $p_{0}$ is by definition

$$
F=\left\{x \in H_{\mathbb{C}}^{2}: d\left(x, p_{0}\right) \leq d\left(x, \gamma p_{0}\right), \forall \gamma \in \Gamma\right\} .
$$

The group $\Gamma$ acts discretely if and only if $F$ has nonempty interior, and, in that case, $F$ is a fundamental domain for $\Gamma$ as long as no element of the group fixes the point $p_{0}$. If $p_{0}$ is fixed, then one only gets a fundamental domain modulo the action of the finite group that fixes it.

Note that $F$ is bounded by a number of hypersurfaces of equidistance, also called bisectors. Even though their definition makes sense in any metric space, it is easy to imagine that these hypersurfaces can be quite pathological, in particular, they need not be smooth or connected in general. Here, we focus on the case of $H_{\mathbb{C}}^{2}$, where Dirichlet domains were already considered in [Giraud 21] (see also [Mostow 80, Goldman 99]).

In practice, it is quite difficult to verify whether or not $F$ is nonempty and, if so, to determine its combinatorics. It is not even clear whether it has finitely many faces (in fact, this is not true in general, even for some very elementary groups, see [Goldman and Parker 92]).

We wish to study cases where $F$ only has finitely many faces, so that we can actually write the polyhedron as

$$
F=F_{W}=\left\{x \in H_{\mathbb{C}}^{2}: d\left(x, p_{0}\right) \leq d\left(x, \gamma p_{0}\right), \forall \gamma \in W\right\},
$$

for some finite subset $W \subset \Gamma$.

The main difficulties can be summarized as follows:

1. How do we find an appropriate set $W$ ?

2. Given a finite set $W \subset \Gamma$, how do we determine the combinatorics of $F_{W}$ ?

3. Does $F_{W}$ have side pairings, and does it satisfy the cycle conditions of the Poincaré polyhedron theorem (see Section 7)?

Part (1) is usually very tedious, but it can be done by computer experimentation (a detailed procedure is given in [Deraux 05]).

For the Mostow groups, one easily obtains a guess for reasonable candidates for the sets $W$ (but one needs to be very cautious, the guess taken in [Mostow 80] seems reasonable, but it is not always correct, as we shall discuss in Section 11).

Part (2) is the most difficult. We shall describe experimental methods for doing this with a computer, but it turns out that it is extremely difficult to prove the results rigorously.

Assuming Part (2) has been done correctly, Part (3) is very straightforward (most of the verifications follow automatically from the fact that $F_{W}$ is obtained by a Dirichlet construction). 


\section{ELEMENTARY PROPERTIES OF BISECTORS}

Dirichlet polyhedra are bounded by hypersurfaces equidistant between two points in complex hyperbolic space. These are not totally geodesic and can have fairly complicated intersection patterns. We review some of the results in [Mostow 80] and [Goldman 99].

Definition 4.1. The bisector between two distinct points $p_{0}$ and $p_{1}$ in $H_{\mathbb{C}}^{2}$ is the set of points that are equidistant from $p_{0}$ and $p_{1}$ :

$$
\mathcal{B}\left(p_{0}, p_{1}\right)=\left\{x \in H_{\mathbb{C}}^{2}: d\left(x, p_{0}\right)=d\left(x, p_{1}\right)\right\}
$$

The formula for the distance between two points was given in Equation (2-2). We shall always rescale the vectors $p_{j}$ so that $\left\langle p_{0}, p_{0}\right\rangle=\left\langle p_{1}, p_{1}\right\rangle$, and the equation of the bisector then becomes simply

$$
\left|\left\langle x, p_{0}\right\rangle\right|=\left|\left\langle x, p_{1}\right\rangle\right|
$$

In order to get points inside complex hyperbolic space, we consider only negative vectors $x \in \mathbb{C}^{3},\langle x, x\rangle<0$. It is sometimes useful, however, to study the solution set in all of $\mathbb{C}^{3}$, or rather in projective space $P_{\mathbb{C}}^{2}$. The corresponding solution set is then (a special case of) an extor (see [Goldman 99] for the definition).

\subsection{Slice Decomposition}

The solution set of the equation $\left|\left\langle x, p_{0}\right\rangle\right|=\left|\left\langle x, p_{1}\right\rangle\right|$ is obviously foliated by complex lines $\left\langle x, p_{0}\right\rangle=\mu\left\langle x, p_{1}\right\rangle$, for complex numbers $\mu$ with $|\mu|=1$.

Equivalently, each solution in $\mathbb{C}^{3}$ is on a unique complex plane of the form $v_{\mu}^{\perp}$, where

$$
v_{\mu}=p_{0}-\mu p_{1},|\mu|=1
$$

Equation (4-3) parametrizes a circle $\sigma$, which splits into two $\operatorname{arcs} \sigma^{-}$and $\sigma^{+}$consisting of vectors of negative or positive norm, respectively.

The $v_{\mu}^{\perp}$, for $v_{\mu} \in \sigma^{+}$, yield a foliation of the bisector by complex geodesics in the ball, called the slice decomposition. It is easy to check that any complex geodesic contained in the bisector must be a leaf of that foliation.

For each vector $v_{\mu}$, there is a unique vector $v_{\nu}$ that is orthogonal to it (it is clear that the only $\nu$ that works is given by $\left.\nu=\left\langle p_{0}, p_{0}-\mu p_{1}\right\rangle /\left\langle p_{1}, p_{0}-\mu p_{1}\right\rangle\right)$. In particular, the $\operatorname{arc} \sigma^{-}$is itself contained in the bisector, and by construction it is contained in the complex geodesic $\Sigma$ spanned by $p_{0}$ and $p_{1}$. Now, the intersection of $\Sigma$ with $\mathcal{B}$ is obviously a real geodesic, equidistant from $p_{0}$ and $p_{1}$.
Definition 4.2. The geodesic $\sigma^{-}$is called the spine of the bisector. The complex geodesic spanned by $p_{0}$ and $p_{1}$ is called its complex spine.

With a little abuse of language, we sometimes refer to the whole circle $\sigma$ as the spine and to the complex projective line containing it as the complex spine. When this causes any confusion, we refer to them as extended (real or complex) spines.

It is clear from the above description that the slices of the bisector are orthogonal to its spine (and to its complex spine). The slice decomposition is usually stated as in Proposition 4.3.

Proposition 4.3. Every bisector is the inverse image of its real spine under orthogonal projection onto its complex spine. The fibers of the projection are called the complex slices of the bisector.

Definition 4.4. A bisector $\mathcal{B}$ is equidistant from a point $x$ if there is a point $y$ such that $\mathcal{B}=\mathcal{B}(x, y)$.

We leave the proof of the following lemma as an exercise for the reader.

Lemma 4.5. Let $\mathcal{B}$ be a bisector with real spine $\sigma$ and complex spine $\Sigma$. Then $\mathcal{B}$ is equidistant from $x$ if and only if $x \in \Sigma \backslash \sigma$.

There is another natural decomposition of bisectors into totally real geodesic subspaces; such a subspace is contained in the bisector if and only if it contains its real spine. Following [Goldman 99], we shall call these totally real subspaces the meridians of the bisector.

\subsection{Ball Coordinates}

The equation of a general bisector in ball coordinates is quadratic in the real and imaginary parts of the coordinates, as can easily be seen from (4-2).

Lemma 4.6. The equation of any bisector $\mathcal{B}$ in ball coordinates is a polynomial of degree at most two in the real and imaginary parts of the coordinates. It is linear if and only if the origin of the coordinates lies on the real spine of $\mathcal{B}$.

From the meridian decomposition, Lemma 4.6 follows from the well-known fact that a Lagrangian is linear if and only if it contains the origin (think of the one-dimensional analogue, saying that geodesics in the 
Poincaré disk are straight lines if and only if they go through the origin).

Hence, given a pair of bisectors, there is no system of coordinates where they both have a linear equation (unless their real spines intersect).

\subsection{Bisectors in Dirichlet Domains}

In view of the discussion in Section 4.2, in order to find the combinatorics of a Dirichlet domain, one must describe the solution set of a number of quadratic inequalities. We shall only consider the cases where we have a finite number of such inequalities. Our approach reduces this problem to a two-dimensional problem, by determining the combinatorics of the various 2 -faces of the domain separately. Such 2 -faces lie on the intersection of two bisectors - we will describe a way to obtain convenient coordinates on the intersection in Section 5 .

The bisectors bounding a Dirichlet domain $F_{W}$ (see Section 3) form a very special family of bisectors; namely, they are all equidistant from a given point $p_{0}$. In view of Lemma 4.5, this means that all their complex spines intersect (Goldman calls such bisectors coequidistant; see [Goldman 99]).

Another important property is the following:

Lemma 4.7. Let $\mathcal{B}_{1}$ and $\mathcal{B}_{2}$ be two coequidistant bisectors. Then $\mathcal{B}_{1} \cap \mathcal{B}_{2}$ cannot contain any totally real totally geodesic subspace.

Proof: Let $\mathcal{B}_{j}=\mathcal{B}\left(p_{0}, p_{j}\right)$. Suppose $L$ is a totally real totally geodesic subspace in the intersection. Then, it contains both real spines; hence, the antiholomorphic involution in $L$ preserves both bisectors, but would have to interchange $p_{0}$ and $p_{j}$, hence, $p_{1}=p_{2}$.

The intersection of coequidistant bisectors can certainly contain a complex geodesic, however.

Lemma 4.8. Let $\mathcal{B}_{1}$ and $\mathcal{B}_{2}$ be two coequidistant bisectors, whose intersection contains a complex geodesic. Then, their complex spines are equal, and their real spines intersect.

Proof: The only complex geodesics contained in a bisector are its slices [Goldman 99]. Suppose the two bisectors contain a complex geodesic $S$. By the slice decomposition, $S$ is orthogonal to both complex spines. Since the complex spines have a point in common, they must coincide (otherwise one would get a geodesic triangle whose angles add up to more than $\pi$ ). The fact that the real spines intersect follows immediately from the slice decomposition (see Proposition 4.3).

The following result is crucial to the study of Dirichlet domains.

Theorem 4.9. Let $\mathcal{B}_{1}$ and $\mathcal{B}_{2}$ be two coequidistant bisectors with distinct complex spines. Then their intersection is a smooth nontotally geodesic disk that is contained in precisely three bisectors.

If $\mathcal{B}_{j}=\mathcal{B}\left(p_{0}, p_{j}\right), j=1,2$, then the third bisector is of course $\mathcal{B}\left(p_{1}, p_{2}\right)$. The fact that there are no other bisectors containing $\mathcal{B}_{1} \cap \mathcal{B}_{2}$ is due to Giraud, and the fact that the intersection is connected was proved by Goldman [Goldman 99].

Definition 4.10. The intersection of two coequidistant bisectors with distinct complex spines is called a Giraud disk. We also say that the two bisectors have Giraud intersection.

We summarize the previous result as follows. The intersection of two coequidistant bisectors, if nonempty, is either a complex totally geodesic disk or a Giraud disk.

As we shall see in the next section by exhibiting explicit coordinates, if the intersection is Giraud, then the equations $\left|\left\langle x, p_{0}\right\rangle\right|=\left|\left\langle x, p_{1}\right\rangle\right|=\left|\left\langle x, p_{2}\right\rangle\right|$ define a torus in projective space, referred to as a Clifford torus in [Goldman 99]. We sometimes call it a Giraud torus, since it extends a Giraud disk. Note that it is the intersection in projective space of the two extors that extend the bisectors $\mathcal{B}_{j}$ (see [Goldman 99] for more on extors).

The following result refines the statement that bisectors in complex hyperbolic space are not totally geodesic (for a proof see [Mostow 80] or [Goldman 99]).

Lemma 4.11. Let $q_{1}$ and $q_{2}$ be two distinct points on the bisector $\mathcal{B}=\mathcal{B}\left(p_{0}, p_{1}\right)$. The geodesic between $q_{1}$ and $q_{2}$ is contained in $\mathcal{B}$ if and only if these points are in some slice or meridian of $\mathcal{B}$.

In fact, Dirichlet domains, which are intersections of half spaces bounded by bisectors, are not convex (even though they are clearly star-shaped with respect to the center of the domain).

Definition 4.12. For $\gamma \in \Gamma$, we write $\widehat{\gamma}$ for the bisector

$$
\widehat{\gamma}=\mathcal{B}\left(p_{0}, \gamma^{-1} p_{0}\right)
$$


Remark 4.13. Note that we use the same definition as Mostow, in order to make it easier to compare with the original text [Mostow 80]. Most references mentioning Dirichlet domains would probably call this bisector $\widehat{\gamma^{-1}}$ instead.

It is obvious from the definition that $\alpha$ sends $\widehat{\alpha}$ to $\widehat{\alpha^{-1}}$, and more generally

$$
\alpha(\widehat{\alpha} \cap \widehat{\beta})=\widehat{\alpha^{-1}} \cap \widehat{\beta \alpha^{-1}} .
$$

Note that this relation becomes much more natural when naming the bisectors in the usual way; see Remark 4.13.

Consider a Dirichlet domain $F_{W}$ as in (3-2). For a given $\gamma \in W$, note that $\widehat{\gamma} \cap F_{W}$ need not be a topological ball in general (see [Deraux 05] for examples).

As a consequence, one needs to define faces carefully, namely, by taking the open faces to be the connected components of the interior of $\widehat{\gamma} \cap F_{W}$ in $\widehat{\gamma}$.

With "faces" interpreted in that manner, one proves just as in Lemma 3.3.2 of [Mostow 80] that the faces are topological balls. Note, however, that Mostow's Lemma 3.3.1 (which claims that for any $\gamma \in W$, the intersection of any complex slice of the bisector $\widehat{\gamma}$ intersects $F_{W}$ in a convex polygon) is incorrect.

\section{EXPERIMENTAL METHODS}

\subsection{Spinal Coordinates on Bisector Intersections}

5.1.1 Cospinal bisectors. The bisectors $\mathcal{B}\left(p_{0}, p_{1}\right)$ and $\mathcal{B}\left(p_{0}, p_{2}\right)$ have the same complex spine if and only if $p_{0}$, $p_{1}$, and $p_{2}$ are represented by linearly dependent vectors in $\mathbb{C}^{3}$.

It follows from the slice decomposition (see Proposition 4.3) that two cospinal bisectors intersect if and only if their real spines intersect, in a point $p$. In that case, their intersection consists of a complex geodesic $S$, which is perpendicular to their common complex spine through $p$.

We then choose a basis $\left\{v_{1}, v_{2}\right\}$ for (the lift to $\mathbb{C}^{3}$ of) $S$, with $\left\langle v_{1}, v_{2}\right\rangle=0,\left\langle v_{1}, v_{1}\right\rangle=-1$, and $\left\langle v_{2}, v_{2}\right\rangle=1$, so that a nice coordinate on $S$ is obtained by writing its vectors as $v_{1}+z v_{2},|z|<1$. The intersection $S \cap \mathcal{B}\left(p_{0}, p_{3}\right)$ with a third bisector has an equation of the form

$$
\left|\left\langle v_{1}+z v_{2}, p_{0}\right\rangle\right|=\left|\left\langle v_{1}+z v_{2}, p_{3}\right\rangle\right|,
$$

which is a (possibly degenerate) circle in the $z$-plane. In particular, the intersection of $S$ with a number of half spaces is bounded by circles or lines in the plane, which we consider an easy problem (even though one needs to be careful with issues of precision). One should be aware that such an intersection need not be connected in general, since the arcs of circles bounding it are not necessarily geodesics.

5.1.2 Giraud disks. We now explain a convenient way to parameterize Giraud intersections. Consider two coequidistant bisectors $\mathcal{B}\left(p_{0}, p_{1}\right)$ and $\mathcal{B}\left(p_{0}, p_{2}\right)$, which we assume not to be cospinal (i.e., the three points $p_{j}$ are represented by linearly independent vectors in $\mathbb{C}^{3}$ ).

We dehomogenize the coordinates on the affine chart given by the complement of $p_{0}^{\perp}$. In other words, we use $y=x /\left\langle x, p_{0}\right\rangle$ as coordinates, and these three coordinates satisfy $\left\langle y, p_{0}\right\rangle=1$.

The bisector intersection is then given by

$$
\begin{aligned}
& \left|\left\langle y, p_{1}\right\rangle\right|=1, \\
& \left|\left\langle y, p_{2}\right\rangle\right|=1 .
\end{aligned}
$$

We simply take $u_{1}, u_{2}$ as complex coordinates on the ball, where $u_{j}=\left\langle y, p_{j}\right\rangle$, so that the Giraud disk is the intersection with the ball of the torus

$$
\left|u_{1}\right|=\left|u_{2}\right|=1
$$

in projective space. We refer to $u_{1}$ and $u_{2}$ as spinal coordinates.

Note that complex hyperbolic space will not be given by the unit ball in the coordinates $\left(u_{1}, u_{2}\right)$. In fact, the intersection of the torus with $H_{\mathbb{C}}^{2}$ can be obtained as follows. Let $P$ be the matrix whose columns are the coordinates of the vectors $p_{j}$, so that we have

$$
\begin{array}{r}
y^{T} H \bar{P}=\left[1, u_{1}, u_{2}\right]=U^{T}, \\
\langle y, y\rangle=U^{T}\left(P^{T} H \bar{P}\right)^{-1} \bar{U} .
\end{array}
$$

The last expression can be rewritten in the form

$$
a_{0}+2 \mathfrak{R e}\left\{a_{1} u_{1}+\left(a_{2}+a_{3} \bar{u}_{1}\right) u_{2}\right\},
$$

where the $a_{j}$ are easily obtained from the Hermitian matrix $\left(P^{T} H \bar{P}\right)^{-1}$. Observe that $a_{0}$ is real (it comes from the diagonal entries of the Hermitian matrix $P^{T} H \bar{P}$ ), whereas the other $a_{j}$ are complex, in general.

The trace on the torus $\left|u_{1}\right|=\left|u_{2}\right|=1$ of the boundary of the ball can obtained by solving a family of quadratic equations

$$
\left\{\begin{array}{l}
2 \mathfrak{R e}\{\alpha z\}=\beta \\
|z|=1
\end{array}\right.
$$

where

$$
\begin{aligned}
& \alpha=\alpha\left(u_{1}\right)=a_{2}+a_{3} \bar{u}_{1}, \\
& \beta=\beta\left(u_{1}\right)=-a_{0}-2 \mathfrak{R e}\left\{a_{1} u_{1}\right\} .
\end{aligned}
$$


It is not obvious, a priori, that the intersection with the ball is connected, but this was proved by Goldman in [Goldman 99]. In other words, there is a unique arc on the circle $\left|u_{1}\right|=1$ for which the equations in (5-3) have a solution (and there are two solutions in the interior of the interval).

It is elementary to see that $(5-3)$ has a solution if and only if

$$
|\beta| \leq 2|\alpha|
$$

In fact, there is a unique solution if $|\beta|=2|\alpha|$, two solutions if $|\beta|<2|\alpha|$, and infinitely many solutions if $\alpha=\beta=0$.

In particular, the image of the Giraud disk under projection onto the complex spine of $\beta\left(p_{0}, p_{1}\right)$ corresponds to the set of values $u_{1}$ satisfying

$$
\left|a_{0}+a_{1} u_{1}+\bar{a}_{1} \bar{u}_{1}\right|<2\left|a_{2}+a_{3} \bar{u}_{1}\right| \text {. }
$$

After squaring both sides, we obtain expressions of degree 4 in the real and imaginary parts of $u_{1}$. This immediately implies that there can be at most two connected components in the intersection of the bisectors, but coequidistance implies that there is only one [Goldman 99].

It is clear from the description that the circles $u_{1}=$ const correspond to the intersection of the slices of the first bisector with the second, and $u_{2}=$ const to the intersection of the slices of the second bisector with the first bisector. The slices of the third bisector $\mathcal{B}\left(p_{1}, p_{2}\right)$ are easily seen to intersect the torus in "lines of slope one," namely, $u_{2}=\mu u_{1},|\mu|=1$.

Remark 5.1. The above three families of curves, given by the intersection with the ball of the horizontal, vertical, and slope 1 slices of the Clifford torus, can be proved to be hypercycles in the Poincaré disk of the appropriate slice of the respective bisectors (see [Mostow 80] or [Goldman 99, Section 7.3]). We shall not need this fact in the present paper; all we need is the fact that they are arcs of circles.

\subsection{Exploring the 2-Faces}

In order to understand the combinatorics of intersections of half-spaces bounded by bisectors, we want to study all of its 2-faces separately. Given the intersection $S$ of two given bisectors, we want to determine the intersection of $S$ with a number of half spaces bounded by finitely many other bisectors.

Our strategy is to plot on $S$ the trace of all the other bisectors and to consider the connected components of the complement of the union of these curves. We shall give some explicit examples in Section 6, but for now we start with some general considerations. We focus on the case where $S$ is a Giraud disk, the cospinal case being much easier to handle (see Section 5.1.1).

Using spinal coordinates $u_{1}$ and $u_{2}$ on $S=\mathcal{B}\left(p_{0}, p_{1}\right) \cap$ $\mathcal{B}\left(p_{0}, p_{2}\right)$, we write the equation of another bisector $\mathcal{B}\left(p_{0}, p_{3}\right)$ as

$$
\left|\delta_{0}+\delta_{1} u_{1}+\delta_{2} u_{2}\right|=1 \text {. }
$$

The coefficients $\delta_{j}$ are obtained as follows. Remembering that we normalize our coordinates so that $\left\langle y, p_{0}\right\rangle=1$, the coefficients $\delta_{j}$ are simply defined by the relation

$$
p_{3}=\bar{\delta}_{0} p_{0}+\bar{\delta}_{1} p_{1}+\bar{\delta}_{2} p_{2}
$$

This makes sense because we assumed that the vectors $p_{0}, p_{1}$, and $p_{2}$ are independent and have the same norm. Note that there is some ambiguity since we could multiply $p_{1}, p_{2}$, or $p_{3}$ by complex numbers of absolute value 1 , but this simply results in shifting the coordinates $u_{j}$ by multiplication by a complex number of absolute value 1 .

Along each slice where $u_{1}$ is constant, the corresponding values of $u_{2}$ are obtained by solving equations exactly as in (5-3), now with

$$
\begin{aligned}
\alpha & =\overline{\left(\delta_{0}+\delta_{1} u_{1}\right)} \delta_{2}, \\
\beta & =1-\left|\delta_{0}+\delta_{1} u_{1}\right|^{2}-\left|\delta_{2}\right|^{2}
\end{aligned}
$$

Once again, for a given $u_{1}$, such an equation has $0,1,2$, or infinitely many solutions.

Away from the degenerate cases, one obtains an explicit parameterization for the intersection $S \cap \mathcal{B}\left(p_{0}, p_{3}\right)$, of the form

$$
u_{2}=\frac{\beta \pm \sqrt{\beta^{2}-4|\alpha|^{2}}}{2 \alpha},
$$

with $\alpha$ and $\beta$ depending on $u_{1}$ as in (5-8). This parameterization is only valid on the locus of $\left|u_{1}\right|=1$, where the discriminant $\Delta=\beta^{2}-4|\alpha|^{2}$ in (5-9) is strictly negative (which could consist of up to two arcs on the circle).

The locus where $\Delta>0$ is irrelevant, and the behavior of the curve $S \cap \mathcal{B}\left(p_{0}, p_{3}\right)$ on the locus $\Delta=0$ is readily analyzed (there is either a unique solution for $u_{2}$ or $u_{2}$ is arbitrary). In the next section, we shall describe one possible computer program that plots these curves, for instance, by using log coordinates $t_{j}$, where $u_{j}=e^{2 \pi i t_{j}}$. The only difficulty is to handle the degenerate situations - note that the only problem with our parameterization is the presence of "vertical lines" in the graphs. The cases where $u_{2}$ is arbitrary for a given value of $u_{1}$ are easy to detect in Equation (5-6). They correspond to having either $\delta_{2}=0$ or $\left|\delta_{2}\right|=1$ and $u_{1}=-\delta_{0} / \delta_{1}$ 
(a geometric interpretation of these two cases is given in [Deraux 05]).

\section{Remark 5.2.}

1. There is, of course, a similar parameterization corresponding to solving for $u_{1}$ in terms of $u_{2}$. This gives another way to handle the vertical lines that could possibly appear in the $u_{1}$-parameterization. It is easy to understand when there are horizontal or vertical lines on the graph in terms of the coefficients $\delta_{j}$ of $(5-6)$.

2. Consider a case where a vertical line occurs at some $u_{1}$, but there are two distinct values of $u_{2}$ for nearby values of $u_{1}$. It can be checked (see [Deraux 05]) that the parameterizations (5-9) have a limit on both sides, so that dividing by numbers close to 0 does not cause too much trouble in the results.

\subsection{Description of a Computer Program}

The java applet [Deraux 04a] provides a convenient way to explore the combinatorics of the 2-faces of Dirichlet domains for the Mostow groups (or for any group given by an explicit set of generators). From there, one can deduce the combinatorics of the whole Dirichlet domain, at least conjecturally.

There are, of course, two cases, depending on whether the 2-face is totally geodesic or not. This is done by computing the determinant of $P=\left[p_{0}, p_{1}, p_{2}\right]$, and if the determinant is close to zero, we consider the bisectors as being cospinal (in [Deraux 04a] we use double precision and consider the bisectors cospinal if $\left.|\operatorname{det} P|^{2}<10^{-10}\right)$.

If the bisectors are (at least close to being) cospinal, we draw a number of circles on the unit disk, which is an easy task - we shall not worry about issues of precision here, and consequently we give no claim of rigor in the combinatorics of our Dirichlet domain.

If the bisectors have Giraud intersection, we use spinal coordinates and produce a plot in log coordinates - recall that we write $u_{j}=e^{2 \pi i t_{j}}$. In our program [Deraux 04a], we split the interval of values of $t_{1} \in[a, b]$ into 500 equal pieces, and for each value of $t_{1}$ in the grid, we check whether $\Delta\left(u_{1}\right)<0$, and, if it is, we use Equation (5-9) to plot the corresponding two points on the graph.

The main problem with the above is that we might be missing some vertical lines in the graph. The simplest way to handle this (which is implemented in [Deraux 04a]) is to plot the graph a second time, using $u_{2}$ as the parameter instead of $u_{1}$.

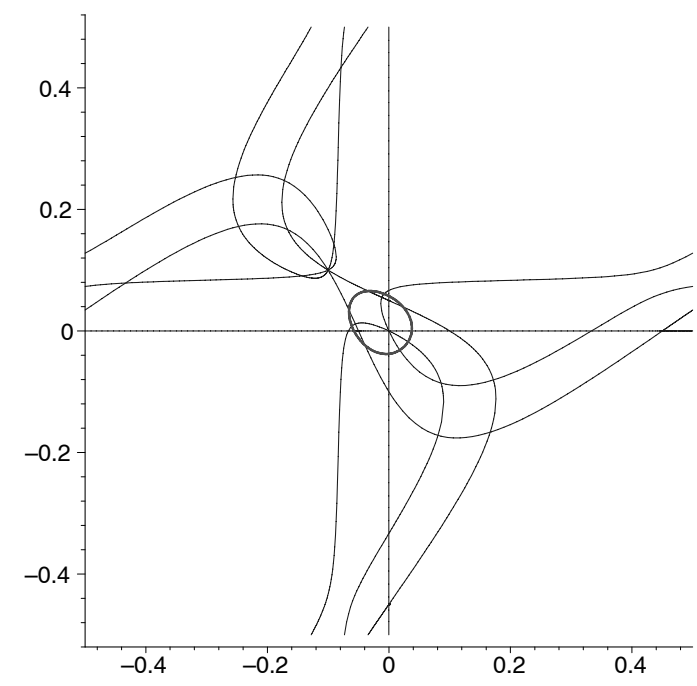

FIGURE 1. The trace of four bisectors on some generic bisector intersection.

Figure 1 shows five curves traced on a torus (identified with a square with real coordinates $t_{1}$ and $t_{2}$, so that $\left.u_{j}=e^{2 \pi i t_{j}}\right)$. The picture was drawn using Maple, for greater precision than in [Deraux 04a] (in fact, the pictures obtained by both methods are practically identical). The thick oval is the intersection with the boundary of the ball, the interior of the ball corresponding to the interior of the oval. The other curves correspond to intersections with four bisectors, two of them having two irreducible components.

\section{DIRICHLET DOMAINS FOR THE FINITE GROUPS}

Recall from Section 2.4 that the groups $\Gamma(p, t)$ contain natural finite subgroups $\Gamma_{i j}$ generated by $R_{i}$ and $R_{j}$. For convenience of notation, we consider the group $\Gamma_{12}$ (the other ones are obtained by conjugation by the isome$\operatorname{try} J)$.

Natural fundamental domains can be obtained from the description in terms of triangle groups given in Section 2.4 , by lifting the sides of the triangle on $\mathbb{P}_{\mathbb{C}}^{1}$ to totally real planes in $H_{\mathbb{C}}^{2}$ (this is the approach taken in [Falbel and Paupert 04]). Such domains will not be Dirichlet domains, however. In fact, we will see that the Dirichlet domains for $\Gamma_{12}$ are much more complicated, and, in particular, they are far from being cones over the fixed point $p_{12}$.

Indeed, recall that the equation for a bisector in nonhomogeneous ball coordinates is, in general, a quadratic equation in the real and imaginary parts of the coordinates (see Section 4.2). The equation reduces to a linear one if and only if the real spine of the bisector goes 
through the origin of the system of nonhomogeneous coordinates.

In particular, if we center the coordinates at the fixed point $p_{12}$ and take a point $p_{0} \neq p_{12}$, none of the bisectors bounding the Dirichlet domain centered at $p_{0}$ will be linear! This explains the perhaps unexpected complexity of the results of this section. We mention that the results stated here differ quite significantly from the claims in [Mostow 80].

Specifically, Mostow claims that for any $p, t$, the fundamental domain $F_{12}$ has only ten faces (which sounds surprising since the groups $\Gamma_{i j}$ have very different orders for different values of $p$ ). The specific set $W_{12}$ of group elements that Mostow uses is

$$
W_{12}=\left\{R_{i}^{ \pm 1},\left(R_{i} R_{j}\right)^{ \pm 1},\left(R_{i} R_{j} R_{i}\right)^{ \pm 1}, i \neq j=1,2\right\}
$$

Recall that the generating reflections braid, i.e., $R_{1} R_{2} R_{1}=R_{2} R_{1} R_{2}$ (see Section 2 ), so that the above set really consists of only ten elements, and not 12 , as might appear at first sight.

It might sound surprising that $(6-1)$ does not vary with $p$ since the orders of the corresponding groups $\Gamma_{i j}$ are different. It is also unclear whether the set of relevant words should remain the same when $t$ changes (the groups $\Gamma_{i j}$ for various values of $t$ are of course isomorphic, but the position of the mirrors of generating reflections with respect to the center of the Dirichlet domain changes).

Proposition 6.1. For $p=3$, the polyhedron $F_{12}$ has at least 18 faces, and the group elements of the form $\left(R_{i} R_{j} R_{i}^{-1}\right)^{ \pm 1}$ and $R_{i} R_{j}^{-1}$ need to be included in order to obtain a fundamental domain.

The conjectural combinatorics of $F_{12}$, for small phase shift, i.e., $|t|<\frac{1}{2}-\frac{1}{p}$, are given in Figures 6 and 7 (compare with the pictures on page 220 of [Mostow 80]). Note that the precise combinatorics are conjectural, even though the fact that a certain face is nonempty can easily be proved rigorously. We only list the case $p=3$ and small phase shift for the sake of brevity, and because we are interested not so much in the finite groups but rather in the corresponding lattices.

Our pictures are obtained using the experimental method sketched in Section 5, without worrying about the dependence on the phase shift parameter (we fix a particular set of braiding reflections by fixing a value of the phase shift $\varphi$ ).

\subsection{Examples of 2-Faces}

We now describe some examples of $\widehat{\alpha_{1}} \cap \widehat{\alpha_{2}} \cap F_{12}$, where $F_{12}$ is the Dirichlet domain for the finite group $\Gamma_{12}$ centered at the barycenter of the mirrors of the three reflections $R_{1}, R_{2}$, and $R_{3}$, namely, $p_{0}=[1,1,1]^{T}$.

These sets can be fairly complicated, and, in particular, it is not clear whether or not they are connected.

Definition 6.2. The connected components of the set $*\left(\widehat{\alpha_{1}} \cap \widehat{\left.\alpha_{2}\right)}=\right.$

$$
\begin{aligned}
\left\{x \in \widehat{\alpha_{1}} \cap \widehat{\alpha_{2}}: d\left(x, p_{0}\right)\right. & <d\left(x, \gamma p_{0}\right), \\
\forall \gamma & \left.\in \Gamma_{12}, \gamma \neq 1, \alpha_{1}^{-1}, \alpha_{2}^{-1}\right\}
\end{aligned}
$$

are called open 2-faces of the polyhedron $F_{12}$.

It turns out that all sets in (6-2) in the Dirichlet domains for the groups that appear in [Mostow 80] are connected, so we shall not bother giving different names to the connected components. Note that, in general, however, these sets are not connected (for examples, see [Deraux 05]).

As we shall see in the examples below, the closure of that open 2-face is not necessarily equal to

$$
\begin{aligned}
\widehat{\alpha_{1}} \cap \widehat{\alpha_{2}} \cap F_{12}=\left\{x \in \widehat{\alpha_{1}} \cap \widehat{\alpha_{2}}: d\left(x, p_{0}\right) \leq d\left(x, \gamma p_{0}\right),\right. \\
\left.\forall \gamma \in \Gamma_{12}\right\},
\end{aligned}
$$

which can have lower-dimensional components.

6.1.1 A cospinal example. We start with the easier case, where the two bisectors $\widehat{\alpha_{1}}$ and $\widehat{\alpha_{2}}$ are cospinal (in which case their intersection is simply a complex line $L$ ). Recall that this is equivalent to saying that $p_{0}, p_{1}=$ $\alpha_{1}^{-1} p_{0}$, and $p_{2}=\alpha_{2}^{-1} p_{0}$ are linearly dependent.

Recall from the discussion in Section 5 that the intersection of a bisector $\widehat{\gamma}$ with the complex line $L$ is an arc of a circle (except for degenerate situations, where $L$ is a complex slice of $\widehat{\gamma}$ ). Once again, we mention that it can be proved that this arc is actually a hypercycle (see Remark 5.1 and [Goldman 99]).

We discuss the example $\widehat{R_{1}} \cap \widehat{R_{1}^{-1}}$ in some detail. Since $R_{1}$ is a complex reflection, this intersection is simply the mirror of $R_{1}$. The corresponding 2-face $\widehat{R_{1}} \cap \widehat{R_{1}^{-1}} \cap F_{12}$ is a sector bounded by two geodesics for small phase shift, and it is slightly more complicated for large phase shift (see Figures 2 and 3).

The point $p_{12}$ is fixed by the whole group $\Gamma_{12}$, so that all the corresponding bisectors go through a given point, which we take to be the origin of the disk coordinates. 


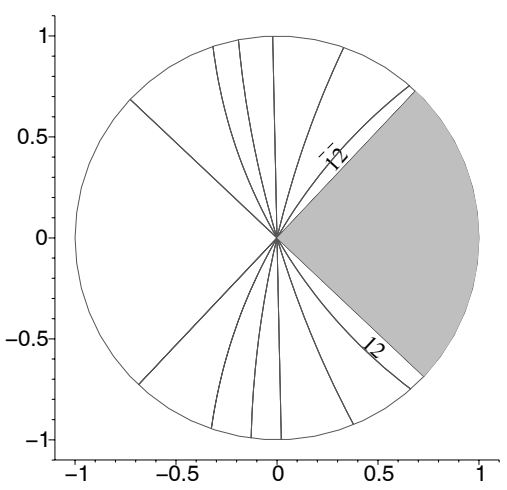

FIGURE 2. The 2-face of $F_{12}$ on the mirror of $R_{1}$, for $p=3$ and $t=1 / 18$.

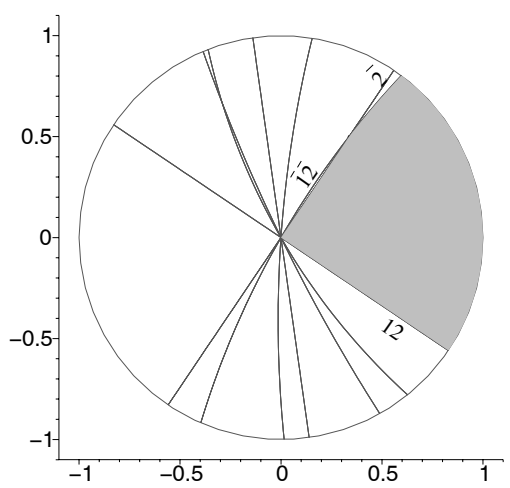

FIGURE 3. The 2 -face of $F_{12}$ on the mirror of $R_{1}$, for $p=3$ and $t=11 / 30$.

Remark 6.3. The pictures given here can be obtained with the applet [Deraux 04a], but the reader should be aware that the notation for bisectors is opposite from the one used in the present paper (see Remark 4.13). In the applet, $\widehat{\gamma}$ stands for $\mathcal{B}\left(p_{0}, \gamma p_{0}\right)$ rather than $\mathcal{B}\left(p_{0}, \gamma^{-1} p_{0}\right)$.

6.1.2 2-faces on Giraud disks. If $p_{0}, p_{1}=\alpha_{1}^{-1} p_{0}$, and $p_{2}=\alpha_{2}^{-1} p_{0}$ are linearly independent, then the intersection $\widehat{\alpha_{1}} \cap \widehat{\alpha_{2}}$ is not totally geodesic, but, as discussed in Section 4, it has natural coordinates and we can easily plot the various intersections $\widehat{\alpha_{1}} \cap \widehat{\alpha_{2}} \cap F_{12}$ using a computer.

We discuss a couple of examples of 2-faces that illustrate the main difficulties. The shaded region in Figure 4 is the open 2 -face $*\left(\widehat{R_{1}} \cap \widehat{R_{1} R_{2} R_{1}}\right)$. In order to obtain such a picture, we start by plotting all intersections with $\widehat{\gamma}$ in spinal coordinates $u_{1}, u_{2}$ (in the picture we actually use $t_{1}$ and $t_{2}$, where $\left.u_{j}=e^{2 \pi i t_{j}}\right)$. This cuts the disk $\widehat{R_{1}} \cap \widehat{R_{1} R_{2} R_{1}}$ into a number of regions, and it is a bit tedious, but not particularly difficult, to test which ones are in the intersection of all the appropriate half-spaces $\gamma^{+}$.

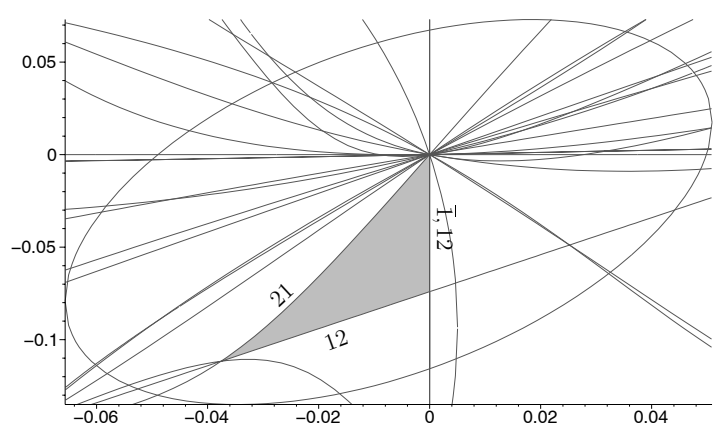

FIGURE 4. The open 2 -face $*\left(\widehat{R_{1}} \cap \widehat{R_{1} R_{2} R_{1}}\right)$, for $p=3$, $t=1 / 18$.

One striking point is that $\widehat{R_{1}} \cap \widehat{R_{1} R_{2} R_{1}} \cap F_{12}$ consists of the closure of the open face $*\left(\widehat{R_{1}} \cap \widehat{R_{1} R_{2} R_{1}}\right)$, together with an edge. Indeed, the whole ray from the origin down along the vertical axis in the picture is contained in $F_{12}$, which is, in fact, contained in the intersection of the five bisectors corresponding to $R_{1}, R_{1}^{-1}, R_{1} R_{2}, R_{1} R_{2} R_{1}$, and $R_{1} R_{2} R_{1}^{-1}$.

This should be only partly surprising, since we already analyzed the intersection of these bisectors in $\widehat{R_{1}} \cap \widehat{R_{1}^{-1}}=$ $e_{1}^{\perp}$ and found that it was indeed a ray that goes all the way to the boundary of the ball (see Figure 2).

We draw one more neighboring face, namely, ${ }^{*} \widehat{\left(R_{1}\right.} \cap$ $\left.\widehat{R_{1} R_{2}}\right)$. Once again, $\widehat{R_{1}} \cap \widehat{R_{1} R_{2}} \cap F_{12}$ contains a segment that is not in the closure of the open face (but that is actually part of the closure of the previous open face

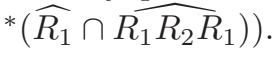

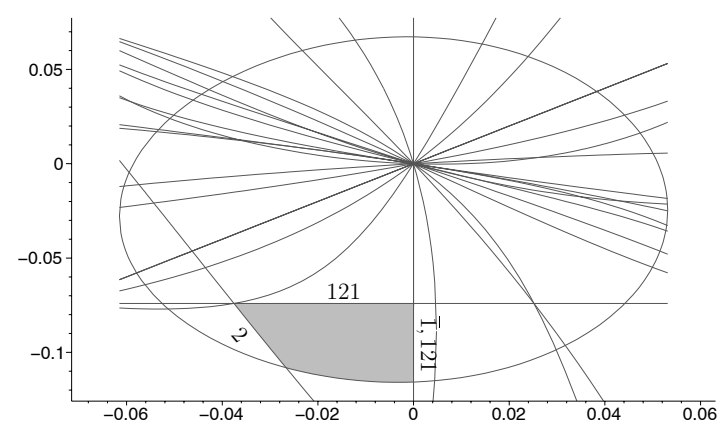

FIGURE 5. The open 2 -face $*\left(\widehat{R_{1}} \cap \widehat{R_{1} R_{2}}\right)$, for $p=3$, $t=1 / 18$.

\subsection{Description of all the 2-Faces}

It is quite clear that the methods from the previous paragraph allow one to describe all the possible 2-faces of the fundamental domain, by repeating the above procedure for all possible pairs of bisectors $\widehat{\alpha_{1}} \cap \widehat{\alpha_{2}}$ in the group (or 


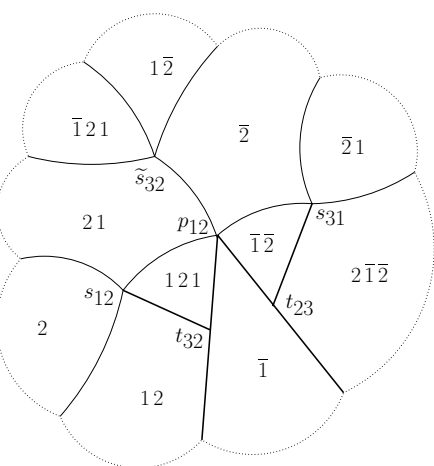

(a) $\widetilde{R_{1}}$

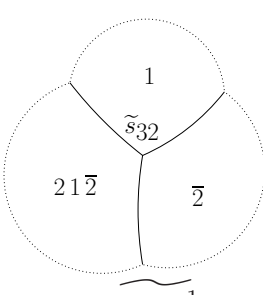

(b) $\widetilde{R_{1} R_{2}^{-1}}$
FIGURE 6. The combinatorics of some faces of $F_{12}$, for small phase shift $(t=1 / 18)$. On each 2 -face, we write a shortcut notation for the neighboring face $\widehat{\gamma}$, using $i$ and $\bar{i}$ for $R_{i}$ and $R_{i}^{-1}$, respectively.

rather in a well-chosen finite set of elements in the group). In this manner, one gets a proof of Proposition 6.1.

There are of course issues of precision, and we do not attempt to prove the results rigorously. The main point is that it is fairly easy to prove that a given 2 -face is nonempty, but it is tedious to prove that the precise combinatorics are as they seem from experimentation.

Figures 6 and 7 describe the results for $p=3$ and $t=1 / 18$. It is not clear that the same combinatorics

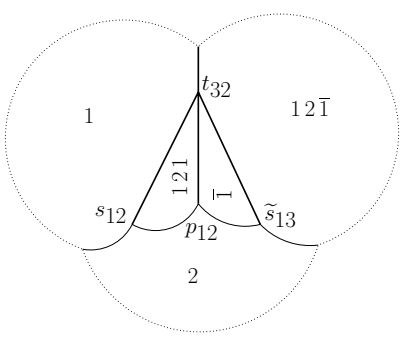

(a) $\widetilde{R_{1} R_{2}}$

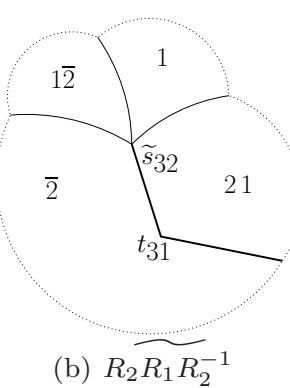

(b) $\overline{R_{2}} \overline{R_{1} R_{2}^{-1}}$

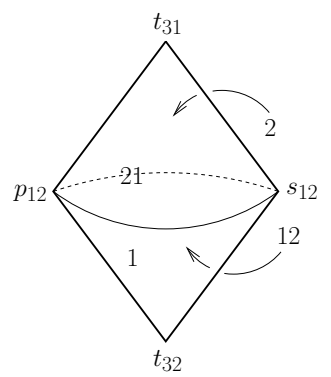

(c) $\widetilde{R_{1} R_{2} R_{1}}$

FIGURE 7. The combinatorics of some faces of $F_{12}$, for small phase shift $(t=1 / 18)$. The dotted lines are contained in the boundary of the ball, whereas the dashed ones have a different meaning. One edge is dashed in (c) simply to indicate that it is hidden behind another 2 -face. hold for any small phase shift, i.e., whenever $-\left(\frac{1}{2}-\frac{1}{p}\right)<$ $t<\left(\frac{1}{2}-\frac{1}{p}\right)$, or for different values of $p$. In fact, one can see (cf. Section 11) that, for $p=5$, the combinatorics are not stable on the intervals corresponding to large phase shift, $|t|>\frac{1}{2}-\frac{1}{p}$.

For the sake of saving space, we only show some faces that are representative of all the combinatorial possibilities. It is easy to deduce the combinatorics of the other faces by symmetry (see Section 8).

Finally, we mention that these figures correct the pictures given on page 220 of [Mostow 80], and that the domains are not cones over the point $p_{12}$.

\section{THE POINCARÉ POLYHEDRON THEOREM}

The most convenient way to transform our experimental results into rigorous proofs would be to use the appropriate version of the Poincaré polyhedron theorem.

Let $F=F_{W}$ be the polyhedron in $H_{\mathbb{C}}^{2}$ bounded by the bisectors $\hat{w}=\mathcal{B}\left(p_{0}, w^{-1} p_{0}\right), w \in W$. Suppose $W$ is symmetric (i.e., $w^{-1} \in W$ whenever $w \in W$ ), and $w$ yields a side pairing transformation

$$
w: F \cap \hat{w} \rightarrow F \cap \widehat{w^{-1}},
$$

so that the image of the polyhedron $F$, under the side pairing $w$, intersects $F$ along one face precisely, with no further intersection.

In what follows, we shall sometimes write $\widetilde{w}$ for the face of $F$ on the bisector $\hat{w}$, i.e.,

$$
\widetilde{w}=F \cap \hat{w},
$$

although the notation is somewhat ambiguous since $\tilde{w}$ depends on the poolyhedron $F$.

Since the side pairing $w$ sends $\widetilde{w}$ to $\widetilde{w^{-1}}$, we write this condition as

$$
F \cap w(F)=\widetilde{w^{-1}} .
$$

The following construction gives the cycle transformation attached to each codimension 2 face, usually referred to as "edges." Let $e_{0}$ be such an edge. It is contained in the intersection $\widetilde{v_{0}} \cap \widetilde{w_{0}}$ of two faces for a unique choice of $v_{0}, w_{0} \in W$. Consider the image of the edge under the side pairing corresponding to one of these two transformations, say $v_{0}$. Since it is a codimension 2 face of $F$, we can write it as

$$
e_{1}=v_{0}\left(e_{0}\right)=\widetilde{v_{1}} \cap \widetilde{w_{1}},
$$

for some group elements $v_{1}, w_{1} \in W$. Note that one of these two, say $w_{1}$, is simply $v_{0}^{-1}$. 
Consider the image of $e_{1}$ under $v_{1}$ and write

$$
e_{2}=v_{1}\left(e_{1}\right)=\widetilde{v_{2}} \cap \widetilde{w_{2}},
$$

for $w_{2}=v_{1}^{-1}$ and $v_{2}$ uniquely determined by Equation (7-4). Repeat this process to define a sequence of group elements $v_{k}, w_{k}, k=1, \ldots, r+1$, until we come back to the edge we started with, i.e.,

$$
e_{r+1}=e_{0} \text {. }
$$

Note that we are certain that this process terminates since our set $W$ is finite.

Definition 7.1. The group element $g_{0}=v_{r} v_{r-1} \ldots v_{1} v_{0}$ is called the cycle transformation associated to the edge $e_{0}=\widetilde{v_{0}} \cap \widetilde{w_{0}}$.

Here, we require that $g_{0}$ be the identity on $e_{0}$ (i.e., this is part of the requirement (7-5)).

The element $g_{0}$ is not quite well defined; in fact, it depends on the choice we made for the first side pairing (we could have chosen $w_{1}$ instead of $v_{1}$ ). It is readily checked that the other choice would simply yield the inverse cycle $g_{0}^{-1}$.

Let $g_{e}$ denote the cycle transformation associated to the edge $e$ of $F$. Suppose that for each edge $e$, the cycle $g_{e}$ has finite order $r_{e}$ and the successive images of $F$ under the transformation $g_{e}$ tile a neighborhood of the edge $e$, overlapping only along faces of the images of $F$ (in particular, the interiors of the images of $F$ should be disjoint).

Theorem 7.2. Under the above assumptions, the group $\Gamma$ generated by $W$ in $P U(2,1)$ is discrete, and $F$ is a fundamental domain for $\Gamma$ modulo the action of the finite subgroup of $\Gamma$ that fixes $p_{0}$.

If no element of $\Gamma$ fixes $p_{0}$, a presentation for $\Gamma$ is given by

$$
\left\langle W \mid g_{e}^{r_{e}}=1\right\rangle,
$$

where the $g_{e}$ are the words defining the cycle transformations as in Definition 7.1.

A proof of this result is sketched in [Mostow 80]; see also [Falbel and Parker 05]. Note that the fact that our faces are not totally geodesic complicates matters quite a bit, not only in proving the theorem but also in verifying whether its hypotheses are satisfied. It is very difficult to prove that two given polyhedra (bounded by bisectors, which are not totally geodesic) intersect precisely along one of their faces, even though, as we mentioned before, it can easily be tested experimentally.

We mention that Giraud's result (Theorem 4.9) is a very useful tool for verifying (7-1). Indeed, it implies that most of the edges (here, we regard totally geodesic edges as being exceptional) are contained in precisely three bisectors, of which only two are equidistant from the center of our Dirichlet domain. Indeed, suppose $e=\widetilde{\alpha} \cap \widetilde{\beta}$ is a generic edge (i.e., the coequidistant bisectors $\widetilde{\alpha}$ and $\widetilde{\beta}$ are not cospinal, or, in other words, $p_{0}, p_{1}=\alpha^{-1} p_{0}$, and $p_{2}=\beta^{-1} p_{0}$ are not on a common complex line). Then $e$ is on precisely three bisectors, namely, $\mathcal{B}\left(p_{0}, p_{1}\right)$, $\mathcal{B}\left(p_{0}, p_{2}\right)$, and $\mathcal{B}\left(p_{1}, p_{2}\right)$. Now, $\alpha(e)$ is also a generic edge, and it is equidistant from $\alpha\left(p_{0}\right), \alpha\left(p_{1}\right)=p_{0}$, and $\alpha\left(p_{2}\right)=\alpha \beta^{-1}\left(p_{0}\right)$.

If the polyhedron is to have side pairings, then, by Theorem 4.9, we need one of its faces to be on the bisector $\widehat{\beta \alpha^{-1}}$. Assuming no group element fixes $p_{0}$, the corresponding side pairing can only be $\beta \alpha^{-1}$, and we can easily check that this maps $\alpha(e)$ to $\beta(e)$. Hence, the cycle for $e$ has length 3 , and its cycle transformation is given by $\beta^{-1}\left(\beta \alpha^{-1}\right) \alpha$, which is the identity.

We emphasize one implication of the above discussion: if a generic face $\widetilde{\alpha} \cap \widetilde{\beta}$ appears in the polyhedron $F$, then the face $\widetilde{\alpha^{-1}} \cap \widetilde{\beta \alpha^{-1}}$ must appear as well (provided that none of these group elements fixes $p_{0}$ ). In particular, this gives a necessary condition for the polyhedron $F_{W}$ corresponding to a set $W$ of group elements to have side pairings, which we refer to as being Giraudclosed.

Definition 7.3. We say that the set $W$ is Giraud-closed if whenever $\alpha, \beta \in W$ and the open 2-face of $F_{W}$ in $\widehat{\alpha} \cap \widehat{\beta}$ is generic and nonempty, we have $\beta \alpha^{-1} \in W$.

This also gives a procedure for finding an appropriate set $W$, by including more and more group elements if necessary until $W$ becomes Giraud-closed (but this procedure might never terminate). For more details on this, see [Deraux 05].

If $W$ is indeed Giraud-closed (this is difficult to verify rigourously), then the verification of the hypotheses of the Poincaré polyhedron theorem can be reduced to verifications about complex geodesic 2 -faces, which is much easier (the corresponding cycle transformations are then complex reflections, and one simply needs to study their angles of rotation). 


\section{SYMMETRIES IN THE FUNDAMENTAL DOMAIN}

\subsection{Basic Symmetry}

The basic symmetry in the fundamental domain comes from the fact that the map

$$
\left[\begin{array}{l}
x_{1} \\
x_{2} \\
x_{3}
\end{array}\right] \mapsto\left[\begin{array}{l}
\overline{x_{2}} \\
\overline{x_{1}} \\
\overline{x_{3}}
\end{array}\right]
$$

is an (antiholomorphic) isometry of complex hyperbolic space. It is readily checked that it conjugates $R_{1}$ into $R_{2}^{-1}$ and $R_{2}$ into $R_{1}^{-1}$. This shows that we only need to consider half of the faces of the fundamental domain.

As in Section 7, we write $\widetilde{\gamma}$ for the face $F_{12} \cap \widehat{\gamma}$ on the bisector $\gamma$. Now, for any $\gamma \in \Gamma_{12}$, the isometry $\gamma$ takes the face $\widetilde{\gamma}$ isometrically into $\widetilde{\gamma^{-1}}$. Once we have the combinatorics of $\widetilde{R_{1}}$, we can deduce the combinatorics of $\widetilde{R_{1}^{-1}}$. These are also isometric to $\widetilde{R_{2}}$ and $\widetilde{R_{2}^{-1}}$, by applying the isometry $\sigma_{12}$.

From $\widehat{R_{1} R_{2}}$, we can also deduce $\left(\widetilde{\left.R_{1} R_{2}\right)^{-1}}\right.$, but this face is not isometric with $\widetilde{R_{2} R_{1}}$, unless $t=0$. It turns out that the combinatorics of $\widetilde{R_{1} R_{2}}$ and $\widetilde{R_{2} R_{1}}$ are the same only when $|t| \leq t_{1}$.

\subsection{Codimension 2 Faces and Cycles}

There are, of course, many isometries between the 2faces, obtained by analyzing the cycles, in the terminology of the Poincaré polyhedron theorem (see Section 7). Since we have introduced some new faces to the list given in [Mostow 80], we include a list of these cycles (valid only for small phase shift).

The face $\widetilde{R_{i}} \cap \widetilde{R_{i}^{-1}}$ is fixed by $R_{i}$, since it is simply the mirror of that complex reflection. The cycle transformation is, of course, the reflection $R_{i}$, and it rotates complex hyperbolic space in the direction normal to its mirror by an angle $2 \pi / p$. The copies of $F_{12}$ under powers of $R_{i}$ tile a neighborhood of that edge by construction, since the bisector $\widehat{R_{i}^{-1}}$ is obtained from $\widehat{R_{i}}$ by applying $R_{i}$. In the finite groups $\Gamma_{i j}$, no other pairs of elements yield cospinal bisectors.

The other cycles correspond to Giraud 2-faces. Recall that such an edge is contained in precisely three bisectors because of Giraud's theorem (see Theorem 4.9, and also the discussion in Section 7); hence, the corresponding cycles will have length 3 .

The cycles yield the following presentation for the finite group:

$$
\Gamma_{i j}=\left\langle R_{i}, R_{j} \mid R_{i}^{p}, R_{i} R_{j} R_{i}=R_{j} R_{i} R_{j}\right\rangle
$$

Indeed, the relation stating that $R_{i}$ has order $p$ comes from the 2-face on the mirror of $R_{i}$, and the braid relation is deduced from certain Giraud 2-faces. For instance, Figure 11 implies that the cycle transformation for the edge $\widetilde{R_{2}^{-1}} \cap\left(R_{1} \widetilde{R_{2} R_{1}}\right)^{-1}$ is given by

$$
\left(R_{1} R_{2} R_{1}\right)\left(R_{1} R_{2}\right)^{-1} R_{2}^{-1}
$$

This transformation is the identity because it fixes a Giraud disk, so we get

$$
R_{1} R_{2} R_{1}=R_{2} R_{1} R_{2}
$$

One verifies that all relations obtained from the cycles in Figures 8-11 are trivial, except for the second and fourth cycles in Figure 8 (both yield a relation equivalent to the braid relation).

The four cycles in Figure 8 correspond to triangular faces with one vertex at $p_{12}$ (having the same combinatorics as in Figure 4). The first two cycles in Figure 8 consist of isometric 2-faces, and so do the last two cycles, but the first and third cycle are not isometric, (unless $t=0)$.

$$
\widehat{\widehat{R_{2}^{-1}} \cap \widehat{R_{2} R_{1}} \stackrel{R_{2} R_{1}}{\longrightarrow} \widehat{\left(R_{1} \widehat{R_{2} R_{1}}\right)^{-1} \cap \widehat{\left(R_{2} R_{1}\right)^{-1}}}{ }^{-1} \stackrel{\left(R_{2} R_{1}\right)^{-1}}{\longrightarrow} \widehat{R_{1} R_{2} R_{1}} \cap \widehat{R_{2} R_{1}}}
$$

FIGURE 8.

The four cycles in Figure 9 correpond to faces that do not contain $p_{12}$, and have the same combinatorics as in Figure 5. Once again there are two isometry types among them, unless $t=0$. 


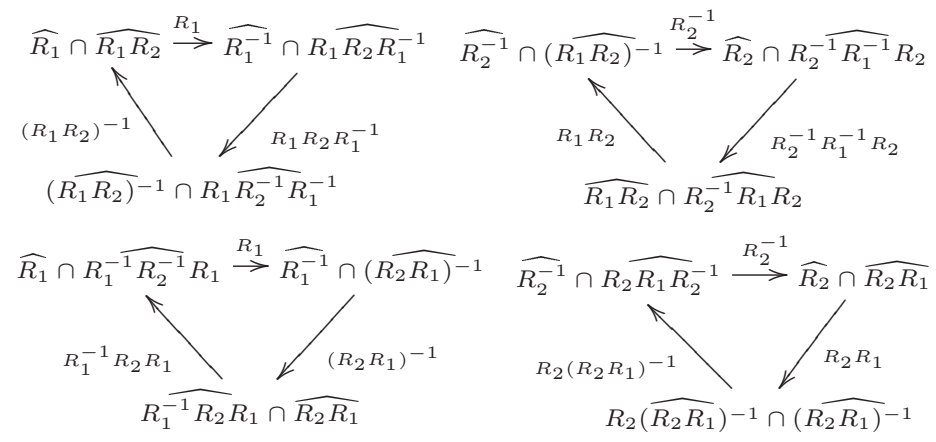

FIGURE 9.

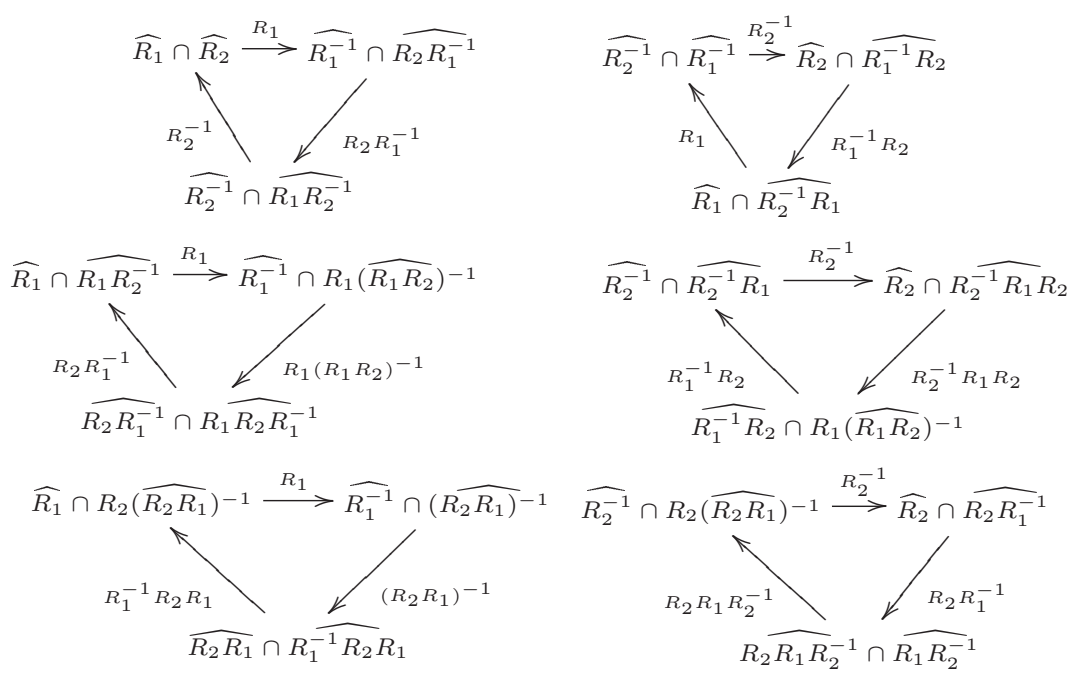

FIGURE 10.

The six cycles in Figure 10 come in isometric pairs. They have one vertex and two (nongeodesic) edges that go off to the boundary.

The 2-faces in the two cycles In Figure 11 have three vertices (one of which is $p_{12}$ ). They are not isometric, unless $t=0$.

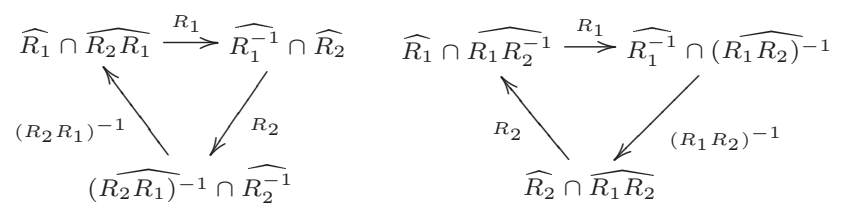

FIGURE 11

\section{LIST OF THE MAIN VERTICES}

We now give the useful list of formulas for the vertices of the fundamental domain that appear in [Mostow 80]. As we shall discuss in Section 11, in some cases there are extra vertices (this happens only for $p=5$ and large phase shift).

For large phase shift, our notation differs slightly from [Mostow 80]; we have chosen to keep the same notation for the vertices for arbitrary phase shift, whereas Mostow describes the vertices $s_{i j}$ by two different formulas (compare with Lemma 12.3 in [Mostow 80]). This should not cause any confusion, since the formulas given below define the points unambiguously, for arbitrary phase shift.

$$
\begin{aligned}
& p_{12}=\left[\xi, \bar{\xi}, 1-\alpha^{2}\right]^{T}, \\
& p_{23}=J p_{12}=\left[1-\alpha^{2}, \xi, \bar{\xi}\right]^{T}, \\
& p_{31}=J p_{23}=\left[\bar{\xi}, 1-\alpha^{2}, \xi\right]^{T},
\end{aligned}
$$

where $\xi=\alpha \varphi\left(1+\alpha \bar{\varphi}^{3}\right)$.

$$
\begin{aligned}
& s_{12}=[\bar{\eta} i \varphi, \bar{\eta} i \bar{\varphi}, 1]^{T}, \\
& s_{23}=J s_{12}=[1, \bar{\eta} i \varphi, \bar{\eta} i \bar{\varphi}]^{T}, \\
& s_{31}=J s_{23}=[\bar{\eta} i \bar{\varphi}, 1, \bar{\eta} i \varphi]^{T},
\end{aligned}
$$




$$
\begin{gathered}
\tilde{s}_{21}=[-\eta i \varphi,-\eta i \bar{\varphi}, 1]^{T}, \\
\tilde{s}_{32}=J \tilde{s}_{21}=[1,-\eta i \varphi,-\eta i \bar{\varphi}]^{T}, \\
\tilde{s}_{13}=J \tilde{s}_{32}=[-\eta i \bar{\varphi}, 1,-\eta i \varphi]^{T}, \\
t_{12}=\left[a(\bar{\varphi}), \overline{a(\bar{\varphi})}, \alpha|a(\bar{\varphi})|^{2}\right]^{T}, \\
t_{21}=\left[\overline{a(\varphi)}, a(\varphi), \alpha|a(\varphi)|^{2}\right]^{T}, \\
t_{23}=J t_{12}=\left[\alpha|a(\bar{\varphi})|^{2}, a(\bar{\varphi}), \overline{a(\bar{\varphi})}\right]^{T}, \\
t_{32}=J t_{21}=\left[\alpha|a(\varphi)|^{2}, \overline{a(\varphi)}, a(\varphi)\right]^{T}, \\
t_{31}=J t_{23}=\left[\overline{a(\bar{\varphi})}, \alpha|a(\bar{\varphi})|^{2}, a(\bar{\varphi})\right]^{T}, \\
t_{13}=J t_{32}=\left[a(\varphi), \alpha|a(\varphi)|^{2}, \overline{a(\varphi)}\right]^{T},
\end{gathered}
$$

where $a(x)=\bar{x}\left(1-\eta i x^{3}\right)$.

$$
\begin{aligned}
& v_{123}=[-\eta i \bar{\varphi}, 1, \bar{\eta} i \varphi]^{T}, \\
& v_{231}=[\bar{\eta} i \varphi,-\eta i \bar{\varphi}, 1]^{T}, \\
& v_{312}=[1, \bar{\eta} i \varphi,-\eta i \bar{\varphi}]^{T}, \\
& v_{321}=[\bar{\eta} i \bar{\varphi}, 1,-\eta i \varphi]^{T}, \\
& v_{132}=[-\eta i \varphi, \bar{\eta} i \bar{\varphi}, 1]^{T}, \\
& v_{213}=[1,-\eta i \varphi, \bar{\eta} i \bar{\varphi}]^{T} .
\end{aligned}
$$

For small phase shift (i.e., $|t|<1 / 2-1 / p$ ), the fundamental domain for the finite group $\Gamma_{12}$ has 11 vertices, given by the $p_{12}, s_{i j}, \tilde{s}_{i j}, t_{13}, t_{31}, t_{23}, t_{32}$. When the hypotheses of the Poincaré theorem hold, the fundamental domain for $\Gamma$ is the intersection of the three fundamental domains for the finite groups $\Gamma_{i j}$ and has 15 vertices given by the first 15 of the above formulas.

The following formulas are quite useful, as they give a partial explanation of the change of behavior in the fundamental domains for the groups as the phase shift is increased beyond its critical value, namely, $|t|=\frac{1}{2}-\frac{1}{p}$.

$$
\begin{aligned}
\left\langle s_{12}, s_{12}\right\rangle & =1-\frac{\cos (\pi t)}{\sin \left(\frac{\pi}{p}\right)}, \\
\left\langle v_{312}, v_{312}\right\rangle & =1+\frac{\cos \left(\frac{2 \pi}{p}-\pi t\right)}{\sin \left(\frac{\pi}{p}\right)}, \\
\left\langle v_{321}, v_{321}\right\rangle & =1+\frac{\cos \left(\frac{2 \pi}{p}+\pi t\right)}{\sin \left(\frac{\pi}{p}\right)} .
\end{aligned}
$$

One checks directly that $s_{12}$ is a negative vector if and only if $|t|<\frac{1}{2}-\frac{1}{p}, v_{312}$ is negative for $t<-\left(\frac{1}{2}-\frac{1}{p}\right)$, and $v_{321}$ is negative for $t>\frac{1}{2}-\frac{1}{p}$ [Mostow 80, page 228].

For completeness we mention that

$$
\begin{aligned}
&\left\langle t_{32}, t_{32}\right\rangle=2(1\left.-\cos \left(\frac{\pi}{p}+\frac{\pi}{2}+\pi t\right)\right) \\
& \times\left\{1-\frac{\sin ^{2}\left(\frac{\pi}{2 p}+\frac{\pi}{4}+\frac{\pi}{2} t\right)}{\sin ^{2}\left(\frac{\pi}{p}\right)}\right\} .
\end{aligned}
$$

This implies that $t_{32}$ is a negative vector if and only if $t>-\left(\frac{1}{2}-\frac{1}{p}\right)$. Similarly, $t_{23}$ is negative if and only if $t<\frac{1}{2}-\frac{1}{p}$.

\section{THE PICARD INTEGRALITY CONDITIONS}

In this section, we review how the cycles of 2 -faces of the Dirichlet domains yield the integrality conditions in Theorem 2.7. These are sometimes called the Picard integrality conditions (they appear in [Picard 81]).

Strictly speaking, the results of this section are valid only for $p=3$ and 4 , since for $p=5$ the Dirichlet domain has additional faces (see Section 11). Note, however, that the analysis of the cycles for complex geodesic 2-faces remains the same for $p=5$, and all differences arise from generic 2-faces only (for which the only discreteness condition is that $W$ be Giraud-closed, see Section 7).

The figures in this section are included mainly for completeness, most of them being already in [Mostow 80] (except for Figure 12(d), which turns out to be crucial in [Deraux et al. 05]). There are several difficulties about proving the results, but the numerical methods above give an easy way to test the claims experimentally.

Remark 10.1. The Dirichlet domains for the groups $\Gamma=$ $\Gamma(p, t)$ turn out to be the intersection of the Dirichlet domains for the three finite groups $\Gamma_{i j}$ generated by any two of the three generators

$$
F=F_{12} \cap F_{23} \cap F_{31} .
$$

We have seen in Section 6 that the Dirichlet domains for the finite groups have many more faces than was stated in [Mostow 80], and it is perhaps surprising that the domains for the full lattices end up differing very little from Mostow's. This is explained by the fact that many of the extra faces for the finite groups are far from the fixed point of the corresponding finite group (see Figures 6 and 7 ).

Since the group $G$ generated by the reflections $R_{i}$ together with the symmetry $J$ is slightly more natural than $\Gamma$ itself, we describe a fundamental domain $F_{G}$ for $G$. This involves decomposing the domain $F$ into three cells, images under $J$ of one another. A natural fundamental domain for the action of $J$ is the sector in complex hyperbolic space bounded by two bisectors $B_{1}=B\left(p_{12}, p_{13}\right)$ and $B_{2}=B\left(p_{12}, p_{23}\right)$. Their complex spines are given by the mirrors of $R_{1}$ and $R_{2}$, respectively.

Some of the faces of the resulting polyhedron are illustrated in Figure 12, whose pictures are drawn in co- 


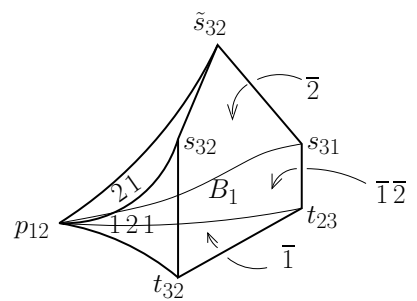

(a) $\widehat{R_{1}} \cap F_{G}$

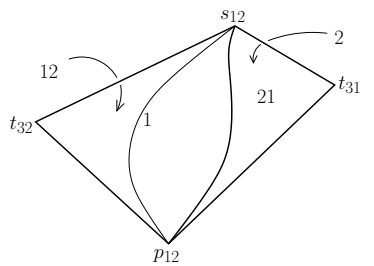

(c) $\widehat{R_{1} R_{2} R}{ }_{1} \cap F_{G}$

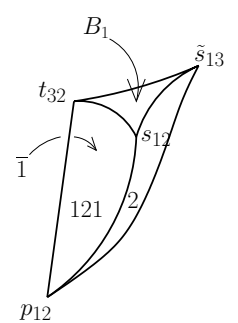

(b) $\widehat{R_{1} R_{2}} \cap F_{G}$

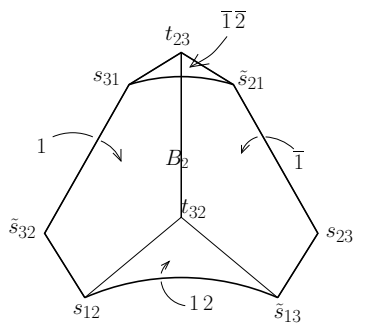

(d) $B_{1} \cap F_{G}$

FIGURE 12. The combinatorics of some faces of the fundamental domain $F_{G}$ for $G$, for $p=3$ and small phase shift $(t=1 / 12)$.

ordinates adapted to each bisector, i.e., so that the spine is along the vertical axis, horizontal planes correspond to complex slices and vertical planes containing the vertical axis yield the meridians. The polyhedron has a total of 12 faces, lying on the bisectors

$$
R_{1}^{ \pm 1}, R_{2}^{ \pm 1},\left(R_{1} R_{2}\right)^{ \pm 1},\left(R_{2} R_{1}\right)^{ \pm 1},\left(R_{1} R_{2} R_{1}\right)^{ \pm 1}, B_{1}, B_{2} .
$$

The side pairings are, of course, given by

$$
\begin{aligned}
R_{1} & : \widehat{R_{1}} \rightarrow \widehat{R_{1}^{-1}} \\
R_{2} & : \widehat{R_{2}} \rightarrow \widehat{R_{2}^{-1}} \\
R_{1} R_{2} & : \widehat{R_{1} R_{2}} \rightarrow \widehat{\left(R_{1} R_{2}\right)^{-1}}, \\
R_{2} R_{1} & : \widehat{R_{2} R_{1}} \rightarrow\left(\widehat{R_{2} R_{1}}\right)^{-1}, \\
R_{1} R_{2} R_{1} & : \widehat{R_{1} R_{2} R_{1}} \rightarrow\left(R_{1} \widehat{R_{2} R_{1}}\right)^{-1}, \\
J & : B_{1} \rightarrow B_{2} .
\end{aligned}
$$

There are three types of totally geodesic 2 -faces, namely, triangles contained in the mirrors of the $R_{j}$ (the bottom face in Figure 12(a)) and triangles contained in slices of the bisectors $\widehat{R_{1} R_{2}}$ and $\widehat{R_{2} R_{1}}$ (the top and bottom faces in Figure 12(d)).

The cycle transformation fixing the mirror of $R_{j}$ is, of course, simply $R_{j}$, and this trivially satisfies the conditions of the Poincaré theorem, since $R_{j}$ rotates by an angle of $2 \pi / p$ in the normal direction (see also the discussion in Section 8).

We now consider the cycles for the geodesic face $\widehat{R_{1} R_{2}} \cap B_{1} \cap F_{G}$. The discussion below is valid for $t>-\left(\frac{1}{2}-\frac{1}{p}\right)$ (otherwise, there is no such 2-face in the fundamental domain).

Lemma 10.2. $\widetilde{R_{1} R_{2}} \cap B_{1}$ is a geodesic triangle on a complex geodesic that is the slice through $t_{32}$ of any of the three bisectors $\widehat{R_{1} R_{2}}, B_{1}$, and $\widehat{\left(R_{3} R_{1}\right)^{-1}}$ (that complex geodesic can also be described as $\left.v_{312}^{\perp}\right)$.

Proof: One checks explicitly that $J^{-1} R_{1} R_{2}=R_{3} R_{1} J^{-1}$ is a complex reflection with mirror $v_{312}^{\perp}$. Its mirror is, by definition, contained in $\widehat{J^{-1} R_{1} R_{2}}=\widehat{R_{1} R_{2}}$ and in $J\left(\widehat{R_{3} R_{1}}\right)^{-1}=\left(\widehat{R_{3} R_{1}}\right)^{-1}$. The last two equalities follow from the fact that $p_{0}$ is fixed by $J$.

One verifies directly that $v_{312}$ is on the extended real spine of $B_{1}$, specifically,

$$
p_{31}+\eta i \varphi p_{12}=\alpha^{2}\left(\eta^{2} \varphi^{2}+\eta i \varphi\right) v_{312},
$$

so that $v_{312}^{\perp}$ is indeed also a slice of $B_{1}$. The fact that $t_{32}$ is on the relevant complex geodesic follows from verifying directly that $\left\langle t_{32}, v_{312}\right\rangle=0$, using the formulas from Section 9 .

Lemma 10.3. $R_{1} R_{2}$ maps $\widetilde{R_{1} R_{2}} \cap B_{1}$ to $\left.\widetilde{\left(R_{1} R_{2}\right.}\right)^{-1} \cap B_{2}$. The cycle transformation for the edge $\widetilde{R_{1} R_{2}} \cap B_{1}$ is $J^{-1} R_{1} R_{2}$.

Proof: One verifies by direct calculation that $R_{1} R_{2}$ maps $t_{32}$ to $t_{13}$. It follows that it maps the slice of 


\begin{tabular}{c|c|c}
2 -face & relation & type \\
\hline$\widehat{R_{i}} \cap \widehat{R_{i}^{-1}}$ & $R_{i}^{p}=1$ & complex \\
$\widehat{R_{2}} \cap \widehat{R_{1} R_{2} R_{1}}$ & $R_{1} R_{2} R_{1}=R_{2} R_{1} R_{2}$ & generic \\
$\widehat{B_{1} \cap B_{2}}$ & $J^{3}=1$ & generic \\
$\widehat{R_{1} R_{2}} \cap B_{1}$ & $\left(J^{-1} R_{1} R_{2}\right)^{k}=1$ & complex \\
$\widehat{R_{2} R_{1}} \cap B_{2}$ & $\left(R_{2} R_{1} J\right)^{l}=1$ & complex
\end{tabular}

TABLE 2. The relations obtained from the cycles of 2faces. The integers $k$ and $l$ are defined by $(10-3)$.

$\widehat{R_{1} R_{2}}$ through $t_{32}$ to the slice of $\widehat{\left(R_{1} R_{2}\right)^{-1}}$ through $t_{13}$, which are precisely the complex lines that appear in Lemma 10.3.

Lemma 10.3 gives a natural necessary condition for $F_{W}$ to be a fundamental domain, by examining the angle of rotation of the reflection $J^{-1} R_{1} R_{2}$.

Lemma 10.4. The reflection $J^{-1} R_{1} R_{2}$ rotates by an angle $2 \pi\left(\frac{1}{4}-\frac{1}{2 p}+\frac{t}{2}\right)$ about its mirror and maps $\widehat{R_{1} R_{2}}$ to $\widehat{\left(R_{3} R_{1}\right)^{-1}}$.

This is done by a direct calculation (computing the eigenvalues of that matrix and multiplying by the appropriate scalar matrix to give it the form in $(2-3))$. The statement about sending one bisector to the other follows from Lemma 10.3 and the fact that $J^{-1}$ maps $\widehat{\left(R_{1} R_{2}\right)}-1$ to $\widehat{\left(R_{3} R_{1}\right)^{-1}}$.

In order for the conditions of the Poincaré polyhedron theorem to hold, one requires the angle in Lemma 10.4 to be of the form $\pi / k$ for some integer $k \in \mathbb{Z}$ (see Section 7 ). This gives one of the integrality conditions in Theorem 2.7.

The second integrality condition comes from the analysis of the cycle transformation for the face $\widetilde{R_{2} R_{1}} \cap B_{2}$ (there is such a face in the domain only if $t<\left(\frac{1}{2}-\frac{1}{p}\right)$ ). One verifies that this cycle transformation is given by the complex reflection $R_{2} R_{1} J$ and that it rotates about its mirror by an angle $2 \pi\left(\frac{1}{4}-\frac{1}{2 p}-\frac{t}{2}\right)$.

Finally, we mention that from the above analysis, one obtains a presentation for the Mostow lattices, by following the various cycles and cycle transformations. The relations are given in Table 2, where we only list some 2 -faces (the relations coming from other faces are all consequences of the ones given in Table 2). Here, we write $k$ and $l$ for the integers that appear in Theorem 2.7, namely,

$$
\begin{aligned}
& \frac{1}{4}-\frac{1}{2 p}+\frac{t}{2}=\frac{1}{k} \\
& \frac{1}{4}-\frac{1}{2 p}-\frac{t}{2}=\frac{1}{l}
\end{aligned} .
$$

\section{VARYING THE PARAMETERS}

As was mentioned above, it is quite difficult to analyze how the combinatorics of the Dirichlet domains change as the parameters $p$ and $t$ vary. We now summarize the results of our experimental observations and show that some modifications are needed in the domains described in [Mostow 80].

\subsection{Summary of Experimental Observations}

We shall write

$$
W_{M}=\left\{R_{i}^{ \pm 1},\left(R_{i} R_{j}\right)^{ \pm 1},\left(R_{i} R_{j} R_{i}\right)^{ \pm 1}\right\},
$$

for the set of words that appears in [Mostow 80]. Our experimental observations suggest the following:

1. for any $p$ and small phase shift, i.e., $|t| \leq \frac{1}{2}-\frac{1}{p}$, $F=F_{W}$ for $W=W_{M}$ and Mostow's combinatorics are correct;

2. for $p<5$ and large phase shift, i.e., $|t|>\frac{1}{2}-\frac{1}{p}$, $F=F_{W}$ for $W=W_{M}$ with combinatorics described in Figure 13;

3. for $p=5$ and large phase shift, $F=F_{W}$ for

$$
W=W_{M} \cup\left\{\left(R_{i} R_{j}\right)^{ \pm 2}\right\}
$$

with different combinatorics for the two relevant values of $t$ (see Figures 14 and 15).

We do not state the preceding results as a theorem, since, in principle, it is conceivable that more faces will be discovered by using a more precise computer program (in the same way that we have found errors in Mostow's analysis by using much more sophisticated computers).

We emphasize the fact that the Dirichlet domains for the groups $\Gamma(5,11 / 30)$ and $\Gamma(5,7 / 10)$ do not have the same combinatorics, even though they both correspond to large phase shift. This differs once again with the analysis given in [Mostow 80], and, in particular, one needs to be extremely cautious about the behavior of Dirichlet polyhedra as parameters vary.

In fact, our experimental methods apply to studying the combinatorics of $F_{W}$ only for a fixed value of $t$, and we shall not attempt to study the stability of the combinatorics as $t$ varies. This is not a big restriction if we want to study the finite number of lattices that arise from this construction (we focus only on the values in Table 1). Note, however, that in order to use these methods for polyhedra corresponding to nondiscrete groups (to construct nonlocally symmetric Kähler manifolds as 


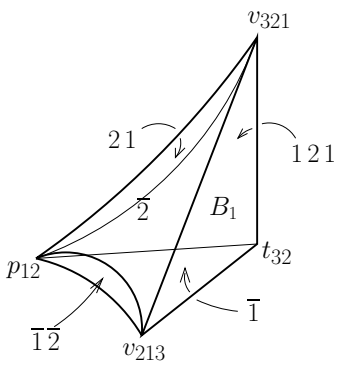

(a) $\widehat{R_{1}} \cap F_{G}$

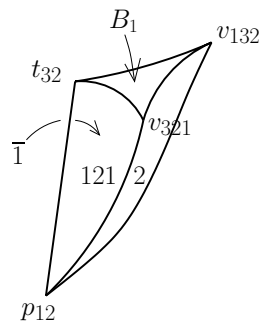

(b) $\widehat{R_{1} R_{2}} \cap F_{G}$

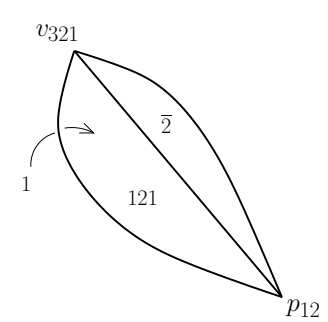

(c) $\widehat{R_{2} R_{1}} \cap F_{G}$

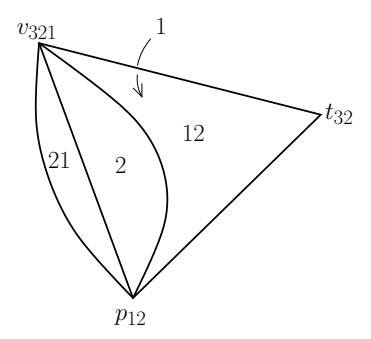

(d) $\widehat{R}_{R_{2} R_{1}} \cap F_{G}$

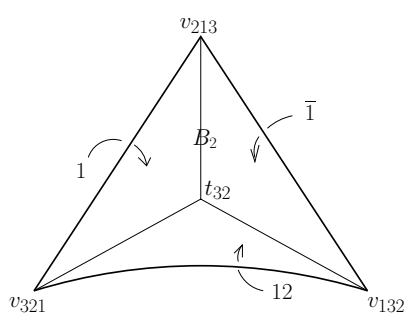

(e) $B_{1} \cap F_{G}$

FIGURE 13. The combinatorics of some faces of the fundamental domain $F_{G}$ for $G$, for $p=3$ and large phase shift $(t=1 / 3)$. Note that we write $i, \bar{i}$ for $R_{i}, R_{i}^{-1}$, respectively.

in [Mostow and Siu 80]), one would have to study this stability question more carefully (in fact, for that purpose, it is more convenient to use the algebro-geometric description of those groups as monodromy groups of hypergeometric functions, see [Deraux 04b] for instance).

When the group satisfies the conditions for discreteness, the Dirichlet polyhedron is only "almost" a fundamental domain for the full group $\Gamma$ generated by $R_{1}, R_{2}$, and $R_{3}$, since in many cases the center $p_{0}$ is fixed by nontrivial elements in the group. It can be checked that the stabilizer of $p_{0}$ is finite and that it is either trivial or cyclic of order 3 , generated by the transformation $J$ (which often turns out to be in the group generated by the reflections $R_{i}$ ).

\subsection{The Case $p=5$, for Large Phase Shift}

In this section, we describe the combinatorics of the Dirichlet domains for $\Gamma(5, t), t>3 / 10$ (a similar analysis holds for $t<-3 / 10$ ). Our domains are "slightly smaller" than the ones described in [Mostow 80], in the sense that we include a few more bisectors to define the Dirichlet polyhedra.

It turns out that the Dirichlet domains for the two discrete groups with large phase shift $(t=11 / 30$ and $7 / 10$ ) have significantly different combinatorics (see Figures 14 and 15). In particular, contrary to what was stated in [Mostow 80], the combinatorics are not inde- pendent of $p$, and there are more cases than simply small and large phase shift.

We describe one specific place where the analysis in [Mostow 80] breaks down for the above two groups and analyze the polyhedron $F_{W}$, where $W$ is as in (11-1), namely,

$$
W=\left\{R_{i}^{ \pm 1},\left(R_{i} R_{j}\right)^{ \pm 1},\left(R_{i} R_{j} R_{i}\right)^{ \pm 1}\right\} .
$$

The error can be seen by experimentation, exploring the combinatorics of 2-face $F_{W} \cap \widehat{R_{1}} \cap \widehat{R_{2}^{-1}}$. For small phase shift, it is bounded by $\widehat{R_{3} R_{1}}, \widehat{R_{2} R_{1}}$, and $\widehat{\left(R_{2} R_{1}\right)^{-1}}$, the last two intersecting in $p_{12}$ (it is obvious that $p_{12}$ is contained in these bisectors, since it is by definition fixed by both $R_{1}$ and $R_{2}$ ). It turns out that more bisectors enter the picture for large phase shift.

Let $c_{21}$ (respectively, $c_{121}$ ) be the curve $\widehat{R_{1}} \cap \widehat{R_{2}^{-1}} \cap$ $\widehat{R_{2} R_{1}}$ (respectively, $\widehat{R_{1}} \cap \widehat{R_{2}^{-1}} \cap \widehat{R_{1} R_{2} R_{1}}$ ). We shall not prove the following experimental observations, but urge the reader to test the claims for themselves, for instance by using the Java application [Deraux 04a] (see Remark 4.13 about notation).

\section{Proposition 11.1.}

1. The curves $c_{21}$ and $c_{121}$ intersect at $p_{12}$ and they are tangent at that point precisely when $t=3 / 10$. 


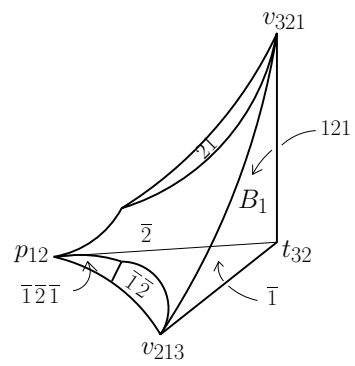

(a) $\widehat{R_{1}} \cap F_{G}$

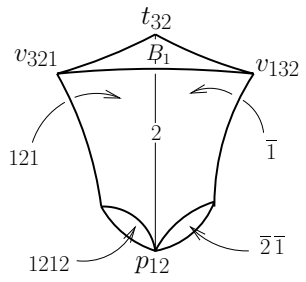

(b) $\widehat{R_{1} R_{2}} \cap F_{G}$

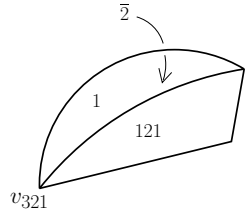

(c) $\widehat{R_{2} R_{1}} \cap F_{G}$

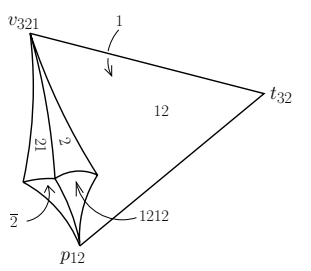

(d) $\widehat{R_{1} R_{2} R_{1}} \cap F_{G}$

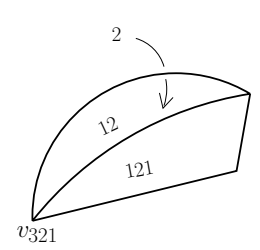

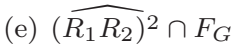

FIGURE 14. Combinatorics of the fundamental domain, for $p=5$ and $t=11 / 30$.

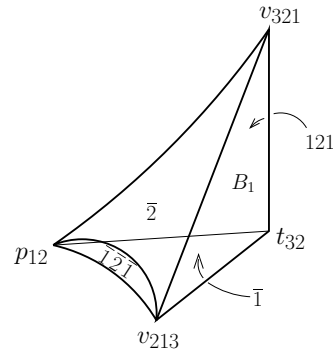

(a) $\widehat{R_{1}} \cap F_{G}$

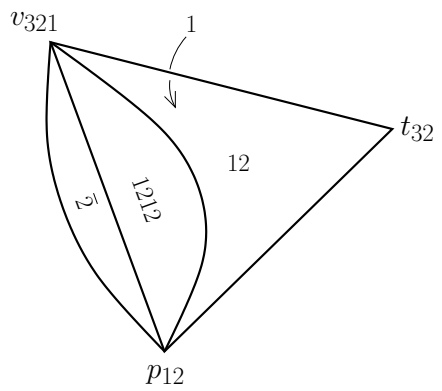

(c) $\widehat{R_{1} R_{2} R_{1}} \cap F_{G}$

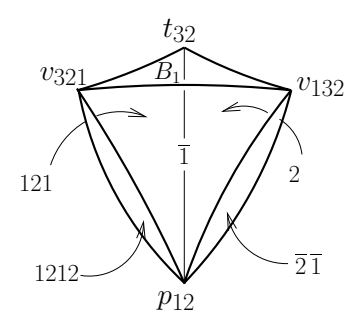

(b) $\widehat{R_{1} R_{2}} \cap F_{G}$

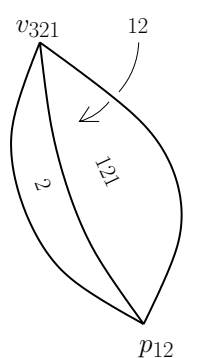

(d) $\left(\widehat{R_{1} R_{2}}\right)^{2} \cap F_{G}$

FIGURE 15. Combinatorics of the fundamental domain, for $p=5$ and $t=7 / 10$. In this case $\widehat{R_{2} R_{3}}=\left(\widehat{\left.R_{3} R_{1}\right)}-1\right.$.

2. For small phase shift, their intersection contains precisely one other point $q_{12}$ approaching $p_{12}$ as $t$ approaches $3 / 10$.

3. For $t=3 / 10$, the curves intersect in the boundary of complex hyperbolic space.

4. For large phase shift, the curves intersect in three points (in the ball) -we still denote by $q_{12}$ the one that is closest to $p_{12}$. The point $q_{12}$ is a vertex of $F_{W}$.

\section{Remark 11.2.}

1. Similar conclusions hold for the curves corresponding to $\left(R_{2} R_{1}\right)^{-1}$ and $\left(R_{1} R_{2} R_{1}\right)^{-1}$. If $t=-3 / 10$, 


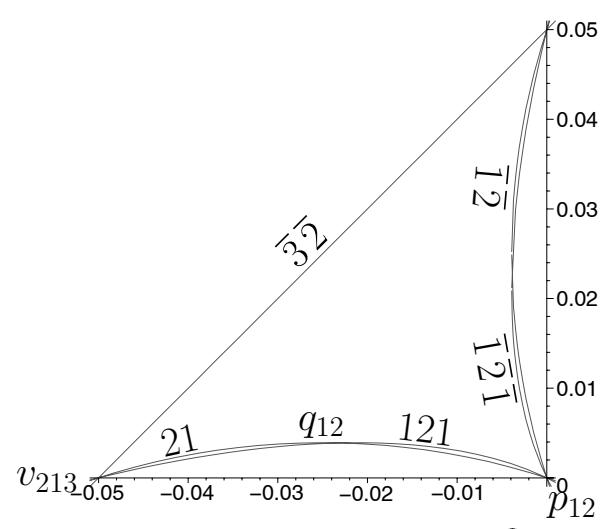

FIGURE 16. The 2 -face $F_{W} \cap \widehat{R_{1}} \cap \widehat{R_{2}^{-1}}$, for $p=5$ and large phase shift $(t=6 / 10)$.

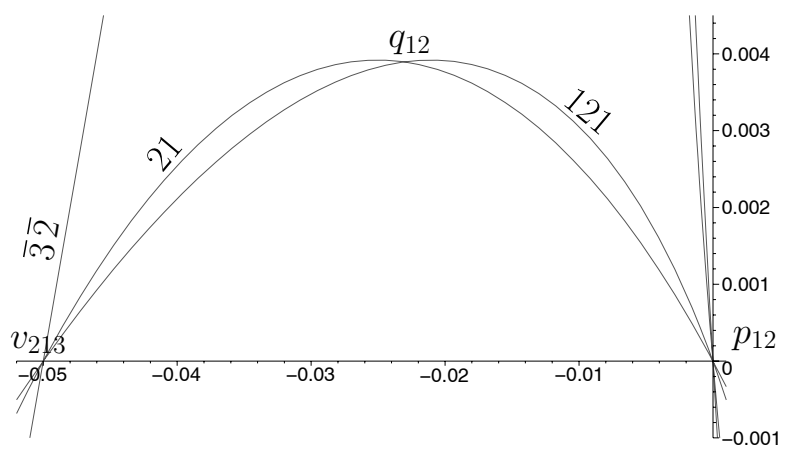

FIGURE 17. Zoomed version of Figure 16.

a similar statement is true for some other 2-face of $F_{W}$, corresponding to $R_{1}^{-1}$ and $R_{2}$.

2. The point $q_{12}$ turns out to be a vertex of the fundamental domain for $t=11 / 30$. When $t=7 / 10$, it coincides with $v_{213}$, where the two curves are again tangent.

3. The curves $c_{21}$ and $c_{121}$ are very close to each other, and, in fact, are barely distinguishable near $p_{12}$ (see Figures 16 and 17)

We now state some consequences of the above changes in the combinatorics of $F_{W}$ as $t$ goes through critical phase shift (the most concrete formulation being Part (2) of Proposition 11.3.

Proposition 11.3. Let $p=5$ and $t>3 / 10$.

1. The polyhedron $F_{W}$ has a nonempty face on the bisector intersection $\widehat{R_{1}} \cap\left(R_{1} \widehat{R_{2} R_{1}}\right)^{-1}$.

2. $R_{1}$ maps $\widehat{R_{1}} \cap\left(R_{1} \widehat{R_{2} R_{1}}\right)^{-1}$ to $\widehat{R_{1}^{-1}} \cap\left(\widehat{\left.R_{1} R_{2}\right)}-2\right.$, and the polyhedron $F_{W}$, where $W$ is as in (11-2), does not have side pairings.
Remark 11.4. Part(1) of Proposition 11.3 can be interpreted as exhibiting some extra intersections between the faces of $F_{W}$, omitted in the description from [Mostow 80]. Note also that both claims in Proposition 11.3 hold only for $p=5$. For $p=3$ or 4 , Mostow's polyhedra seem to be correct.

Proof: Note that (2) follows from (1) together with Giraud's theorem. Indeed, $R_{1}$ maps this bisector intersection to $\widehat{R_{1}} \cap \widehat{\gamma}$, where

$\gamma=\left(R_{1} R_{2} R_{1}\right)^{-1} R_{1}^{-1}=\left(R_{2} R_{1} R_{2}\right)^{-1} \cdot R_{1}^{-1}=\left(R_{1} R_{2}\right)^{-2}$

as follows from (4-4). The latter intersection is contained in precisely three bisectors, only two of which are equidistant from $p_{0}$ (cf. Theorem 4.9).

We claim that $\widehat{\left(R_{1} R_{2}\right)^{-2}}$ is not among the list of bisectors $\widehat{w}, w \in W$. Rather than proving this for general phase shift, we simply mention that, for any fixed value of the phase shift, this claim amounts to verifying that none of 24 group elements fixes $p_{0}$ (namely, the elements $\left.\left(R_{1} R_{2}\right)^{2} w, w \in W\right)$. For $p=5$ and large phase shift, there are two relevant values, $|t|=11 / 30$ and $|t|=7 / 10$.

Part (1) can be checked numerically. For instance, consider the case $t=11 / 30$. We claim that the point $z$ on $\widehat{R_{1}} \cap\left(R_{1} \widehat{R_{2} R_{1}}\right)^{-1}$ with spinal coordinates $u_{j}=e^{2 \pi i t_{j}}$, where $t_{1}=-0.0011$ and $t_{2}=0.0075$, is in the interior of this 2-face of $F_{W}$. This follows from verifying 22 inequalities of the form

$$
\left|\left\langle z, p_{0}\right\rangle\right|<\left|\left\langle z, w^{-1} p_{0}\right\rangle\right|
$$

where $w \in W$ is different from $R_{1}$ and $\left(R_{1} R_{2} R_{1}\right)^{-1}$ (note that $W$ has 24 elements). This can easily be done with the computer.

The proof also suggests an extra set of words that need to be included in order to have side pairings, namely, the $\left(R_{i} R_{j}\right)^{ \pm 2}$ (see the discussion at the end of Section 7 ). Experimentation suggests that in both cases $\Gamma(5,11 / 30)$ and $\Gamma(5,7 / 10)$ no other words need to be included (i.e., that the corresponding set of words is Giraud-closed). However, this depends on combinatorics that are obtained experimentally, and it is conceivable that a more precise computation would yield extra intersections between the various relevant bisectors, forcing us to introduce yet another set of words in order for the polyhedron to have side pairings.

The conjectural combinatorics are summarized in Figures 14 and 15 . 


\section{ACKNOWLEDGMENTS}

The author wishes to thank Elisha Falbel, John Parker, Julien Paupert, Anna Pratoussevitch, Richard Schwartz, and Pierre Will for many useful dicussions on the subject, as well as the referees for their useful comments and suggestions.

\section{REFERENCES}

[Coxeter 67] H. S. M. Coxeter. "Finite Groups Generated by Unitary Reflections." Abh. Math. Sem. Univ. Hamburg 31 (1967), 125-135.

[Deligne and Mostow 86] P. Deligne and G. D. Mostow. "Monodromy of Hypergeometric Functions and Nonlattice Integral Monodromy." Inst. Hautes Études Sci. Publ. Math. 63 (1986), 5-89.

[Deraux 04a] M. Deraux. "Dirichlet Polyhedra for the Mostow Lattices." Java applet. Available from World Wide Web (http://www.math.utah.edu/ deraux/java/ mostow), 2004.

[Deraux 04b] M. Deraux. "On the Universal Cover of Certain Exotic Kähler Surfaces of Negative Curvature." Math. Ann. 329:4 (2004), 653-683.

[Deraux 05] M. Deraux. "Deforming the $\mathbb{R}-$ Fuchsian $(4,4,4)$ Triangle Group into a Lattice." Preprint, 2005.

[Deraux et al. 05] M. Deraux, E. Falbel, and J. Paupert. "New Constructions of Fundamental Polyhedra in Complex Hyperbolic Space." Acta Math. 194 (2005), 155-201.

[Epstein 87] D. B. A. Epstein. "Complex Hyperbolic Geometry." In Analytical and Geometric Aspects of Hyperbolic Space, pp. 93-111, London Math. Soc. Lecture Notes, 11. Cambridge, UK: Cambridge University Press, 1987.

[Falbel and Koseleff 02] E. Falbel and P. -V. Koseleff. "Rigidity and Fexibility of Triangle Groups in Complex Hyperbolic Geometry." Topology 41:4 (2002), 767-786.

[Falbel and Parker 05] E. Falbel and J. Parker. "The Geometry of the Eisenstein-Picard Modular Group." To appear in Duke Math. J., 2005.

[Falbel and Paupert 04] E. Falbel and J. Paupert. "Fundamental Domains for Finite Subgroups of $U(2)$ and
Configurations of Lagrangians." Geom. Dedicata 109 (2004), 221-238.

[Giraud 21] G. Giraud. "Sur certaines fonctions automorphes de deux variables." Ann. Sci. École Norm. Sup. 38 (1921), 43-164.

[Goldman 99] W. M. Goldman. Complex Hyperbolic Geometry, Oxford Mathematical Monographs. Oxford, UK: Oxford University Press, 1999.

[Goldman and Parker 92] W. M. Goldman and J. R. Parker. "Dirichlet Polyhedra for Dihedral Groups Acting on Complex Hyperbolic Space." J. Geom. Anal. 2:6 (1992), $517-554$.

[Mostow 80] G. D. Mostow. "On a Remarkable Class of Polyhedra in Complex Hyperbolic Space." Pacific J. Math. 86 (1980), 171-276.

[Mostow 86] G. D. Mostow. "Generalized Picard Lattices Arising from Half-Integral Conditions." Inst. Hautes Études Sci. Publ. Math. 63 (1986), 91-106.

[Mostow 88] G. D. Mostow. "On Discontinuous Action of Monodromy Groups on the Complex $n$-Ball." J. Amer. Math. Soc. 1 (1988), 555-586.

[Mostow and Siu 80] G. D. Mostow and Y. T. Siu. "A Compact Kähler Surface of Negative Curvature Not Covered by the Ball." Ann. of Math. 112 (1980), 321-360.

[Parker 05] J. Parker. "Cone Metrics on the Sphere and Livne's Lattices." Preprint, 2005.

[Phillips 92] M. Phillips. "Dirichlet Polyhedra for Cyclic Groups in Complex Hyperbolic Space." Proc. Amer. Math. Soc. 115 (1992), 221-228.

[Picard 81] E. Picard. "Sur une extension aux fonctions de deux variables du problème de Riemann relatif aux fonctions hypergéométriques." Ann. ENS 10 (1881), 305-322.

[Schwartz 01] R. E. Schwartz. "Ideal Triangle Groups, Dented Tori, and Numerical Analysis." Annals of Mathematics 153 (2001), 533-598.

[Thurston 98] W. P. Thurston. "Shapes of Polyhedra and Triangulations of the Sphere." Geometry and Topology Monographs 1 (1998), 511-549.

Martin Deraux, Université de Grenoble, Institut Fourier, B.P. 74, 38402 Saint-Martin-d'Hères Cedex, France (deraux@ujf-grenoble.fr)

Received January 26, 2005; accepted June 14, 2005. 